\title{
Treatment of Trypanosoma cruzi with 2-bromopalmitate alters morphology, endocytosis, differentiation and infectivity
}

\author{
Cassiano Martin Batista', Rafael Luis Kessler ${ }^{2,3}$, Iriane Eger $^{4}$ and Maurilio José Soares ${ }^{1 *}$ (D)
}

\begin{abstract}
Background: The palmitate analogue 2-bromopalmitate (2-BP) is a non-selective membrane tethered cysteine alkylator of many membrane-associated enzymes that in the last years emerged as a general inhibitor of protein S-palmitoylation. Palmitoylation is a post-translational protein modification that adds palmitic acid to a cysteine residue through a thioester linkage, promoting membrane localization, protein stability, regulation of enzymatic activity, and the epigenetic regulation of gene expression. Little is known on such important process in the pathogenic protozoan Trypanosoma cruzi, the etiological agent of Chagas disease.

Results: The effect of 2-BP was analyzed on different developmental forms of Trypanosoma cruzi. The $\mathrm{IC}_{50} / 48 \mathrm{~h}$ value for culture epimastigotes was estimated as $130 \mu \mathrm{M}$. The $\mathrm{IC}_{50} / 24 \mathrm{~h}$ value for metacyclic trypomastigotes was $216 \mathrm{nM}$, while for intracellular amastigotes it was $242 \mu \mathrm{M}$ and for cell derived trypomasigotes was $262 \mu \mathrm{M}\left(\mathrm{IC}_{50} / 24 \mathrm{~h}\right)$. Our data showed that 2-BP altered T. cruzi: 1) morphology, as assessed by bright field, scanning and transmission electron microscopy; 2) mitochondrial membrane potential, as shown by flow cytometry after incubation with rhodamine-123; 3) endocytosis, as seen after incubation with transferrin or albumin and analysis by flow cytometry/fluorescence microscopy; 4) in vitro metacyclogenesis; and 5) infectivity, as shown by host cell infection assays. On the other hand, lipid stress by incubation with palmitate did not alter epimastigote growth, metacyclic trypomastigotes viability or trypomastigote infectivity.
\end{abstract}

Conclusion: Our results indicate that 2-BP inhibits key cellular processes of T. cruzi that may be regulated by palmitoylation of vital proteins and suggest a metacyclic trypomastigote unique target dependency during the parasite development.

Keywords: 2-Bromopalmitate, 2-BP inhibition, Differentiation, Endocytosis, Palmitoylation, Trypanosoma cruzi

\section{Background}

Palmitoylation is a post-translational protein modification that consists in addition of palmitic acid to a cysteine residue through a thioester linkage. This modification promotes membrane localization, regulation of enzymatic activity, regulation of gene expression and protein stability [1-3]. Palmitoylation is a reversible, dynamic modification regulated by enzymes that either transfer palmitic acid to a target protein (palmitoyl acyltransferases: PATs) or cleave the thioester linkages between palmitic acid and the modified proteins (palmitoyl protein thioesterases: PPTs) [4]. Palmitoylation represents an important modification

\footnotetext{
* Correspondence: maurilio.soares@fiocruz.br

'Laboratory of Cell Biology, Carlos Chagas Institute/Fiocruz-PR, 81310-020

Curitiba, Paraná, Brazil

Full list of author information is available at the end of the article
}

in cells of a variety of organisms, such as mammals [5-7], yeasts $[8,9]$, fishes $[10]$, plants $[11,12]$ and nematodes [13]. This modification is well characterized in humans, as it is strongly involved in Huntington's disease and other neuropsychiatric diseases [14, 15] and cancer [16]. Palmitoylation was also recorded in pathogenic protozoa, including Toxoplasma gondii [17], Plasmodium falciparum [18] and Trypanosoma brucei [19].

The palmitate analogue 2-bromopalmitate (2-BP) is a non-selective membrane tethered cysteine alkylator of many membrane-associated enzymes that in the last years emerged as a general inhibitor of protein S-palmitoylation [20]. There are two proposed mechanisms for the 2-BP action: direct inhibition of PATs or blockage of palmitic acid incorporation by direct covalent competition with palmitate [21]. It has been suggested that 2-BP also inhibits 
PPTs, disturbing the acylation cycle of the protein GAP-43 at the depalmitoylation level and consequently affecting its kinetics of membrane association [22]. Incubation of the apicomplexan T. gondii with $50 \mu \mathrm{M} 2-\mathrm{BP}$ efficiently altered parasite morphology, gliding and host cell invasion [23]. In the African trypanosome T. brucei, the calculated $\mathrm{IC}_{50}$ values were $197 \mu \mathrm{M}$ for the procyclic form and $226 \mu \mathrm{M}$ for the bloodstream life form [19]. However, no 2-BP or global palmitoylation studies have been reported yet for Trypanosoma cruzi, a protozoan parasite that causes Chagas disease in Latin America.

Two T. cruzi proteins are known to be palmitoylated: TcFCaBP [24], which is involved in parasite motility, and TcPI-PLC [25], which is involved in evading the host immune system. A putative PAT has been identified in this protozoan (TcHIP/TcPAT1), localized in the Golgi complex of different life stages [26] and other nine could be overexpressed in epimastigotes, being mostly located at the anterior end of the parasites [27]. However, other still unidentified proteins should be also palmitoylated in T. cruzi, and palmitoylation is probably involved in diverse biological functions. A recent review on protein acylation in trypanosomatids with a focus on myristoylation and palmitoylation suggested that protein acylation represents an interesting target for the development of new trypanocidal drugs [28]. Indeed, T. cruzi $\mathrm{N}$-myristoyltransferase (TcNMT), an enzyme that catalyzes the attachment of myristic acid to an $\mathrm{N}$-terminal glycine residue of proteins, has been validated as a potential chemotherapeutic target in T. cruzi mammal stages [29].

The aim of this study was to assess the in vitro effect of 2-BP on T. cruzi. The data presented here show that this inhibitor alters the parasite morphology, endocytosis, differentiation and infectivity and suggest the importance of palmitoylation for parasite survival and its involvement in crucial biological processes.

\section{Methods \\ Reagents}

Trypan Blue, Dulbecco's Modified Eagle Medium (DMEM), penicillin (10,000 units), streptomycin (10 mg/ $\mathrm{mL}$ ), trypsin from porcine pancreas, 2-bromopalmitate (2-BP), palmitate, dimethyl sulfoxide (DMSO), potassium chloride, propidium iodide, RNase A, carbonyl cyanide 3-chlorophenylhydrazone (CCCP), acridine orange, Giemsa stain, formaldehyde, paraformaldehyde, glutaraldehyde, Hoechst staining solution and poly-L-lysine were purchased from Sigma-Aldrich (St. Louis, MO, USA). Transferrin-AlexaFluor 633, Albumin-AlexaFluor 488, rhodamine-123, goat anti-mouse IgG AlexaFluor 488 conjugate, goat anti-mouse IgG AlexaFluor 594 conjugate and Prolong Gold were purchased from Molecular Probes/ Life Technologies (Eugene, OR, USA). Potassium ferrocyanide, Permount, sodium cacodylate, osmium tetroxide and PolyBed-812 resin were purchased from Electron Microscopy Sciences (Hatfield, PA, USA). Sodium dodecyl sulfate (SDS) was purchased from Ludwig Biotecnologia (Alvorada, RS, Brazil). Fetal bovine serum (FBS) was purchased from Gibco/Invitrogen/Life Technologies (Eugene, OR, USA). Nonidet 40 (NP-40) was purchased from Anresco Laboratories (San Francisco, CA, USA).

\section{Vero cells}

Vero cells (ATCC CCL-81) isolated from the kidney of the African green monkey Cercopithecus aethiops, were purchased from ATCC (Washington DC, USA). The cells were maintained at $37{ }^{\circ} \mathrm{C}$ in $75-\mathrm{cm}^{2}$ cell culture flasks (Corning Incorporated, Corning, NY, USA) in DMEM medium supplemented with $10 \%$ FBS in a humidified $5 \% \mathrm{CO}_{2}$ atmosphere. For weekly seeding, the cell monolayers were washed twice with PBS, trypsinized and the detached cells were collected by centrifugation for $5 \mathrm{~min}$ at $800 \times g$. The cells were then inoculated at $10^{6}$ cells/flask in fresh DMEM medium and kept as described above.

\section{Trypanosoma cruzi}

Culture epimastigote forms of T. cruzi clone Dm28c, isolated from Didelphis marsupialis in Venezuela [30] were maintained at $28{ }^{\circ} \mathrm{C}$ by weekly passages in Liver Infusion Tryptose (LIT) medium [31] supplemented with $10 \%$ heat-inactivated fetal bovine serum (FBS).

In vitro-derived metacyclic trypomastigotes were obtained by incubating epimastigotes in Triatomine Artificial Urine (TAU/TAU3AAG) medium, according to a previously described metacyclogenesis (i.e., epimastigote -to-trypomastigote differentiation) protocol [32], with a yield of approximately $50 \%$. Metacyclic trypomastigotes were purified with a DEAE-cellulose column as previously described [32].

Cell-derived trypomastigotes were obtained from Vero cell cultures infected with in vitro-derived metacyclic trypomastigotes, at a ratio of 100 parasites/cell. After $4 \mathrm{~h}$ of interaction the host cell monolayers were washed with PBS to remove the non-adherent parasites. Infected cells were then incubated for six days in $10 \mathrm{~mL}$ of DMEM medium supplemented with $10 \%$ FBS, when trypomastigote production peaked. The culture supernatant was collected, and the cell-derived trypomastigotes released into the supernatant were harvested by centrifugation for $15 \mathrm{~min}$ at 3,000 $\mathrm{g}$. The parasites were then used for the experiments and to maintain the infection cycle.

\section{Amplification of PATs genes}

DNA sequences of fourteen TcPATs (PATs 2-15) were identified and used for primer design [27] with subsequent amplification by PCR, using genomic DNA (gDNA) of T. cruzi (Dm28c) epimastigotes. gDNA was 
extracted from three-day-old culture epimastigotes by a phenol-chloroform method [33]. TcPAT1 (TcHIP) primers [26] were also used for PCR. Amplifications were confirmed by $1.0 \%$ agarose gel electrophoresis.

\section{Determination of $\mathrm{IC}_{50}$ value for 2-BP}

Stock solutions at $100 \mathrm{mM}$ of 2-BP and palmitate were prepared in DMSO. The solutions were filtered through a 0.22- $\mu \mathrm{m}$ Millipore filter (Merck Millipore Co, Tullagreen, $\mathrm{CO}$, Ireland) and stored at $4{ }^{\circ} \mathrm{C}$. After dilution in culture medium, the DMSO concentration in the experiments never exceeded $1 \%$, and it did not affect parasite growth.

To calculate the concentration of 2-BP that inhibited $50 \%$ growth of the epimastigote cultures $\left(\mathrm{IC}_{50} / 48 \mathrm{~h}\right)$, the parasites $\left(10^{6} / \mathrm{mL}\right)$ were incubated at $28{ }^{\circ} \mathrm{C}$ with different concentrations of 2-BP ( 25 to $400 \mu \mathrm{M})$ in biological triplicates. Cell counts were made after $48 \mathrm{~h}$ with a Neubauer chamber. The population density was calculated, and the death percentage was estimated relative to the untreated control (LIT medium with 1\% DMSO), generating dose-effect curves. The CompuSyn software [34] was then used to calculate the $\mathrm{IC}_{50} / 48 \mathrm{~h}$ value by using the death percentage for each 2-BP concentration. For morphological analysis, the parasites were processed for bright field, scanning and transmission electron microscopy as described below.

To calculate the $\mathrm{IC}_{50} / 24 \mathrm{~h}$ for metacyclic and cell-derived trypomastigotes, the parasites $\left(10^{6}\right.$ cells $\left./ \mathrm{mL}\right)$ were incubated with different concentrations of 2-BP (0.1 to $175 \mu \mathrm{M}$ for metacyclic trypomastigotes; 0.1 to $400 \mu \mathrm{M}$ for cell-derived trypomastigotes) in biological triplicates. After $24 \mathrm{~h}$ at $28{ }^{\circ} \mathrm{C}$ (or $4 \mathrm{~h}$ at $37{ }^{\circ} \mathrm{C}$ for culture trypomastigotes, to avoid differentiation into extracellular amastigote-like forms) the parasite number was counted in a Neubauer chamber and the death percentage was calculated relative to the untreated control (culture medium with $1 \%$ DMSO). The CompuSyn software was used to calculate the $\mathrm{IC}_{50}$ value.

To calculate the $\mathrm{IC}_{50}$ value for intracellular amastigotes, 24-h-old infected Vero cell cultures $\left(10^{6}\right.$ cells $\left./ \mathrm{mL}\right)$ were incubated with 2-BP $(0.4$ to $125 \mu \mathrm{M})$ in biological triplicates in a humidified $5 \% \mathrm{CO}_{2}$ atmosphere. After 24 and $48 \mathrm{~h}$ of treatment, the infected host cells were lysed by nitrogen decompression [35] and the number of released amastigotes was counted in a Neubauer chamber. Population density was then compared to the untreated control (medium with $0.125 \%$ DMSO). The CompuSyn software was used to calculate the $\mathrm{IC}_{50}$ value. The number of released trypomastigotes was then evaluated after $72 \mathrm{~h}$ of treatment ( $96 \mathrm{~h}$ of infection).

The $\mathrm{IC}_{50} / 24 \mathrm{~h}$ for intracellular amastigotes was calculated with purified amastigotes obtained from 48-h-old infected cells cultures, by lysing the host cells with nitrogen decompression [35]. After three steps of centrifugation at low speed to remove the Vero cells, the purified intracellular amastigotes were incubated at $37{ }^{\circ} \mathrm{C}$ with DMEM medium in 6-well plates (approximately $10^{7}$ parasites/ well) in a humidified $5 \% \mathrm{CO}_{2}$ atmosphere. This cell suspension was centrifuged after 30 min to remove remaining intact Vero cells and new DMEM medium (containing additional 1000 units penicillin and $100 \mu \mathrm{g} / \mathrm{mL}$ streptomycin) was added. The purified isolated intracellular amastigotes were then incubated for $24 \mathrm{~h}$ with 2-BP $(0.4$ to $500 \mu \mathrm{M})$ in biological triplicates. The CompuSyn software was used to calculate the $\mathrm{IC}_{50}$ value.

The 2-BP IC $\mathrm{I}_{50}$ values obtained from the experiments on epimastigote growth and metacyclic trypomastigote viability were used in control assays of lipidic stress, by incubating these forms $\left(48 \mathrm{~h}\right.$ at $28{ }^{\circ} \mathrm{C}$ for epimastigotes, $24 \mathrm{~h}$ at $28{ }^{\circ} \mathrm{C}$ for metacyclic trypomastigoes) with palmitate.

\section{Cytotoxicity to Vero cells}

To calculate the cytotoxicity for Vero cells $\left(\mathrm{CC}_{50} / 24 \mathrm{~h}\right)$, $10^{5}$ cells $/ \mathrm{mL}$ were cultivated in 6-well plates in biological triplicates. After $24 \mathrm{~h}$, the cultures were washed twice with PBS and incubated at $37{ }^{\circ} \mathrm{C}$ with different concentrations of 2-BP (75 to $300 \mu \mathrm{M})$ in a humidified $5 \% \mathrm{CO}_{2}$ atmosphere. After $24 \mathrm{~h}$ of treatment, cell cultures were washed twice with PBS, trypsinized, washed in DMEM medium, stained with Trypan blue $(0.02 \%$ final concentration) for cell viability analysis and counted in Neubauer chamber. The percentage of dead cells was estimated relative to the untreated control (culture medium with $0.3 \%$ DMSO). The CompuSyn software was used to calculate the $\mathrm{CC}_{50} / 24 \mathrm{~h}$ value.

\section{Flow cytometry of $T$. cruzi epimastigotes}

Flow cytometry experiments were performed with a FACS Aria-II (Becton-Dickinson, San Jose, CA, USA). A total of 20,000 events were acquired in the region previously identified as corresponding to $T$. cruzi epimastigotes [36]. The data were analyzed using the FlowJo software package (FlowJo, Ashland, OR, USA). Epimastigotes $\left(2 \times 10^{6}\right.$ cells $)$ were incubated for $48 \mathrm{~h}$ with the $\mathrm{IC}_{50}$ value of $2-\mathrm{BP}(130 \mathrm{uM})$, washed twice with $\mathrm{PBS}$ and then used in the experiments.

For cell cycle assays, epimastigote DNA was stained with propidium iodide (PI). The parasites were incubated with cell cycle buffer $(3.4 \mathrm{mM}$ Tris- $\mathrm{HCl}, 30 \mu \mathrm{g} / \mathrm{mL}$ PI, $0.1 \% \mathrm{NP}-40,10 \mathrm{mM}$ sodium chloride, $700 \mathrm{U} / \mathrm{L}$ RNAse, pH 7.6) and immediately analyzed using a 610/ $20 \mathrm{~nm}$ bandpass filter. Cell debris and doublets were excluded using a width $x$ area gate. The Dean-Jett-Fox algorithm of FlowJo was used to estimate the percentage of cells in the G1, S and G2/M phases of the cell cycle.

For mitochondrial membrane potential analysis, epimastigotes were incubated with $\mathrm{IC}_{50} / 48 \mathrm{~h} 2$ - $\mathrm{BP}$ or palmitate and then incubated for $15 \mathrm{~min}$ at room 
temperature with $10 \mu \mathrm{g} / \mathrm{mL}$ rhodamine-123. After two washes with PBS, the parasites were analyzed using a $530 / 30 \mathrm{~nm}$ bandpass filter. CCCP at $100 \mu \mathrm{M}$ for $5 \mathrm{~min}$ was used as a positive control of the mitochondrial membrane potential destabilization [37]. The relative mitochondrial membrane potential was determined by considering normalized median ratios (treated/control) of the fluorescence level. The normalized fluorescence medians were used for statistical analysis.

For cell viability assay, $\mathrm{IC}_{50} / 48 \mathrm{~h} 2-\mathrm{BP}$ treated epimastigotes were incubated for $5 \mathrm{~min}$ at room temperature with PI $(5 \mu \mathrm{g} / \mathrm{mL})$ and then analyzed without washing. Positively stained cells $(610 / 20 \mathrm{~nm}$ bandpass filter) were considered dead.

For acid compartment analysis, the $\mathrm{IC}_{50} / 48 \mathrm{~h} 2-\mathrm{BP}$ treated epimastigotes were incubated for $15 \mathrm{~min}$ at room temperature with $5 \mu \mathrm{g} / \mathrm{mL}$ acridine orange, washed twice with PBS and then analyzed using a 695/20 nm bandpass filter. The normalized fluorescence medians were analyzed as described above [36]. In parallel, parasites were adhered to glass slides and visualized with epifluorescence microscopy using a B-2A (long pass emission) filter (Nikon, Chiyoda, Japan).

\section{Endocytosis assays}

Epimastigotes were incubated for $4 \mathrm{~h}$ with $\mathrm{IC}_{50} / 48 \mathrm{~h}$ 2-BP, washed twice in PBS and subjected to a previously described endocytosis assay $[35,38]$ using $2 \times 10^{6}$ parasites for flow cytometry analysis or $5 \times 10^{6}$ cells for fluorescence microscopy studies. After 15 min under stress in PBS at $25^{\circ} \mathrm{C}$, the parasites were incubated for $30 \mathrm{~min}$ at $28{ }^{\circ} \mathrm{C}$ with $50 \mu \mathrm{g} / \mathrm{ml}$ transferrin-AlexaFluor 633 or albumin-AlexaFluor 488. Negative control cells were incubated in the absence of labeled transferrin or albumin. For flow cytometry, living epimastigotes were analyzed using $660 / 20$ and $530 / 30 \mathrm{~nm}$ bandpass filters for transferrin-AlexaFluor 633 or albumin-AlexaFluor 488 acquisition, respectively. The normalized median fluorescence intensity of transferrin and albumin was calculated as the ratio between the median fluorescence intensity of the treated and untreated cells. Data acquisition and analysis was performed as described above [35].

\section{2-BP treatment during in vitro metacyclogenesis}

Mid log-phase epimastigotes were collected and submitted to various treatments with $\mathrm{IC}_{50} / 48 \mathrm{~h} 2$ - $\mathrm{BP}$ during metacyclogenesis: (a) Control: five days in LIT/0.13\% DMSO and then incubation for $2 \mathrm{~h}$ in TAU and $72 \mathrm{~h}$ in TAU3AAG media without 2-BP; (b) Pre-stress: five days in LIT medium with $\mathrm{IC}_{50} / 48 \mathrm{~h} 2$-BP followed by incubation for $2 \mathrm{~h}$ in TAU without 2-BP; (c) Stress: five days in LIT medium and then $2 \mathrm{~h}$ in TAU medium containing with $\mathrm{IC}_{50} / 48 \mathrm{~h}$ 2-BP; and (d) Post-stress: five days in LIT medium, stress for $2 \mathrm{~h}$ in TAU medium and incubation for $15 \mathrm{~min}$ in TAU3AAG with $\mathrm{IC}_{50} / 48 \mathrm{~h} 2$-BP.

After 2-BP treatments the cells were washed twice with PBS to remove 2-BP and incubated for $72 \mathrm{~h}$ in TAU3AAG medium. The parasites in the supernatants were then collected and counted in Neubauer chamber to obtain the ratio of metacyclic trypomastigotes / epimastigotes per $\mathrm{mL}$. For morphological analysis, the parasites were processed for light microscopy.

\section{Infection assays}

Metacyclic trypomastigotes obtained from 2-BP treatments during metacyclogenesis (see above) were used to infect Vero cells. Briefly, $10^{7}$ metacyclic trypomastigotes were incubated with $10^{6}$ Vero cells and after $24 \mathrm{~h}$ noninvasive trypomastigotes were removed by PBS washes. After 6 days, the infected cultures were washed with PBS and the released trypomastigotes were collected from the PBS by centrifugation. The trypomastigotes were then counted and processed for light and fluorescence microscopy analysis.

In other experiment, cell-derived trypomastigotes were treated with $\mathrm{IC}_{50} / 4 \mathrm{~h} 2-\mathrm{BP}$ or palmitate and then used to infect Vero cells. Briefly, the parasites $\left(2 \times 10^{7}\right.$ cells $\left./ \mathrm{mL}\right)$ were pre-incubated with $\mathrm{IC}_{50} / 4 \mathrm{~h} 2$-BP or palmitate, washed twice with PBS, counted in Neubauer chamber and then incubated for $2 \mathrm{~h}$ with $10^{6}$ adhered Vero cells. Non-adherent parasites were then washed out with PBS. Four days after infection the released trypomastigotes were collected, counted in Neubauer chamber and processed for light and fluorescence microscopy. Vero cell cultures infected with untreated trypomastigotes were used as a control.

\section{Light microscopy}

The parasites were adhered for $10 \mathrm{~min}$ to $0.1 \%$ poly-L-lysine coated slides, fixed for $2 \mathrm{~min}$ at room temperature with methanol, air dried and then incubated for $30 \mathrm{~min}$ at room temperature with Giemsa stain. The samples were then washed with MilliQ water and mounted with Permount. After $24 \mathrm{~h}$ the slides were observed with a Nikon E600 microscope (100× objective) in bright field mode for image acquisition.

\section{Fluorescence microscopy}

To detect the native TcFCaBP, epimastigotes and metacyclic trypomastigotes were incubated with the respective $\mathrm{IC}_{50}$ values of 2 -BP or palmitate, washed twice in PBS, fixed for $20 \mathrm{~min}$ with $4 \%$ formaldehyde and incubated for $1 \mathrm{~h}$ at room temperature with incubation buffer $(1.5 \%$ BSA/PBS). This step was followed by incubation for $1 \mathrm{~h}$ at $37{ }^{\circ} \mathrm{C}$ with a primary mAb 25 monoclonal antibody against TcFCaBP [39], which was diluted to 1:1000 in incubation buffer. The samples were 
then washed three times in PBS and incubated for $1 \mathrm{~h}$ at $37{ }^{\circ} \mathrm{C}$ with a secondary goat anti-mouse antibody coupled to AlexaFluor-488 or AlexaFluor-594 (1:600) in incubation buffer.

To co-localize transferrin-AlexaFluor-633 or albuminAlexaFluor-488 with cruzipain (CZP), the epimastigotes subjected to the endocytosis assays were fixed for $30 \mathrm{~min}$ in $4 \%$ paraformaldehyde and permeabilized for 5 min with $0.5 \%$ Triton in PBS. For co-localization of $\mathrm{CZP} /$ transferrin-AlexaFluor-633, the samples incubated with CZP-315.D9 $\mathrm{mAb}$ (at $50 \mu \mathrm{g} / \mathrm{ml}$ ) [40], were washed three times in PBS and then incubated with a secondary goat anti-mouse-AlexaFluor-488 antibody (1:600 in incubation buffer). For the co-localization with CZP/albumin-AlexaFluor-488, the samples were incubated with CZP-315.D9 $\mathrm{mAb}$ and then incubated with a secondary goat anti-mouse-AlexaFluor-594 antibody.

After antibody incubations, all samples were washed three times in PBS, incubated for 5 min with $1.3 \mathrm{nM}$ Hoechst 33342, washed twice in PBS and twice in MilliQ Water, mounted with Prolong Gold anti-fading reagent and then examined under a Nikon Eclipse E600 epifluorescence microscope.

\section{Scanning electron microscopy}

Parasites were washed twice in PBS and fixed for $1 \mathrm{~h}$ at room temperature in $2.5 \%$ glutaraldehyde in $0.1 \mathrm{M}$ phosphate buffer, $\mathrm{pH}$ 7.2. The fixed parasites were washed twice in $0.1 \mathrm{M}$ cacodylate buffer and then adhered for $15 \mathrm{~min}$ at room temperature to $0.1 \%$ poly-L-lysine coated coverslips. The non-adhered cells were washed out twice with $0.1 \mathrm{M}$ cacodylate buffer and the adhered cells were then incubated for $15 \mathrm{~min}$ at room temperature with $1 \%$ osmium tetroxide diluted in $0.1 \mathrm{M}$ cacodylate buffer. The samples were washed three times with $0.1 \mathrm{M}$ cacodylate buffer and dehydrated in a graded acetone series $(30 \%, 50 \%, 70 \%, 90 \%$ and $100 \%$ acetone, 5 min each). This step was followed by critical point drying in a Leica EM CPD300 and gold sputtering in a Leica EM ACE200. The samples were visualized in a JEOL JSM-6010 PLUS/LA scanning electron microscope at $20 \mathrm{kV}$.

\section{Transmission electron microscopy}

Parasites were washed twice in PBS and fixed for $1 \mathrm{~h}$ in $2.5 \%$ glutaraldehyde in $0.1 \mathrm{M}$ phosphate buffer at room temperature. The fixed parasites were washed twice in $0.1 \mathrm{M}$ cacodylate buffer and then post-fixed with $1 \%$ osmium tetroxide / $1.6 \%$ potassium ferrocyanide / $5 \mathrm{mM}$ $\mathrm{CaCl}_{2}$ diluted in $0.1 \mathrm{M}$ cacodylate buffer for $30 \mathrm{~min}$ at room temperature. The samples were washed three times with $0.1 \mathrm{M}$ cacodylate buffer, dehydrated in a graded acetone series (5 min in 30, 50, 70, 90 and 100\%) and then embedded in PolyBed812 resin. Ultrathin sections were obtained in a Leica EM UC6 ultramicrotome, collected on copper grids, contrasted with uranyl acetate and lead citrate and then visualized in a JEOL 1400Plus transmission electron microscope at $90 \mathrm{kV}$.

\section{Statistical analysis}

All experiments were performed in independent biological triplicates. Statistical analysis was performed using the software GraphPad Instat (GraphPad Software Inc., La Jolla, CA, USA) for ANOVA followed by a Bonferroni's multiple comparisons test.

\section{Results}

\section{PATs genes in $T$. cruzi}

PCR using specific primers for each gene encoding TcPATs resulted in amplification of fourteen genes with the expected number of base pairs (Additional file 1: Figure S1), except for TcPAT6 (no positive amplification).

\section{Effect of 2-BP on different $T$. cruzi developmental forms} The $\mathrm{IC}_{50} / 48 \mathrm{~h}$ value of 2 -BP for culture epimastigotes was estimated as $130 \mu \mathrm{M}$ (Fig. 1a). The estimated $\mathrm{IC}_{50}$ value $\left(\mathrm{IC}_{50} / 24 \mathrm{~h}\right)$ of 2 - $\mathrm{BP}$ for metacyclic trypomastigotes was $216 \mathrm{nM}$ (Fig. 1b), while for cell-derived trypomastigotes it was $262 \mu \mathrm{M}$ (Fig. 1c).

The cytotoxic effect $\left(\mathrm{CC}_{50} / 24 \mathrm{~h}\right)$ of 2-BP on Vero cells was calculated before the assays with intracellular amastigotes, and its estimated value was $138 \mu \mathrm{M}$. Concentrations higher than $200 \mu \mathrm{M}$ killed $100 \%$ of the host cells. No effect on number and morphology of intracellular amastigotes was observed after 24 or $48 \mathrm{~h}$ of treatment of infected Vero cells with up to $125 \mu \mathrm{M}$ 2-BP (data not shown). However, $125 \mu \mathrm{M}$ 2-BP enhanced the intracellular differentiation into trypomastigote forms.

Isolation of intracellular forms by nitrogen decompression after $24 \mathrm{~h}$ of treatment showed several trypomastigote-like stages, characterized by the presence of a bar kinetoplast close to the nucleus (Additional file 2: Figure S2). There was an increase of approximately $40 \%$ in the number of released trypomastigotes after four days of infection $(72 \mathrm{~h}$ of treatment) when compared to untreated cultures.

Finally, intracellular amastigotes were isolated from the host cells by nitrogen decompression and were then incubated with different concentrations of 2-BP. In this case, the estimated $\mathrm{IC}_{50} / 24 \mathrm{~h}$ value was $242 \mu \mathrm{M}$ (Fig. $1 \mathrm{~d}$ ). This high concentration could explain why we failed to calculate the $\mathrm{IC}_{50}$ value for intracellular amastigotes (no effect up to $125 \mu \mathrm{M})$.

\section{Mislocalization of TcFCaBP after incubation with 2-BP}

Epimastigotes and metacyclic trypomastigotes were treated respectively for 48 or $24 \mathrm{~h}$ with their $2-\mathrm{BP} \mathrm{IC}_{50}$ values and then incubated with a monoclonal antibody against TcFCaBP, a flagellar calcium-binding protein known to be 

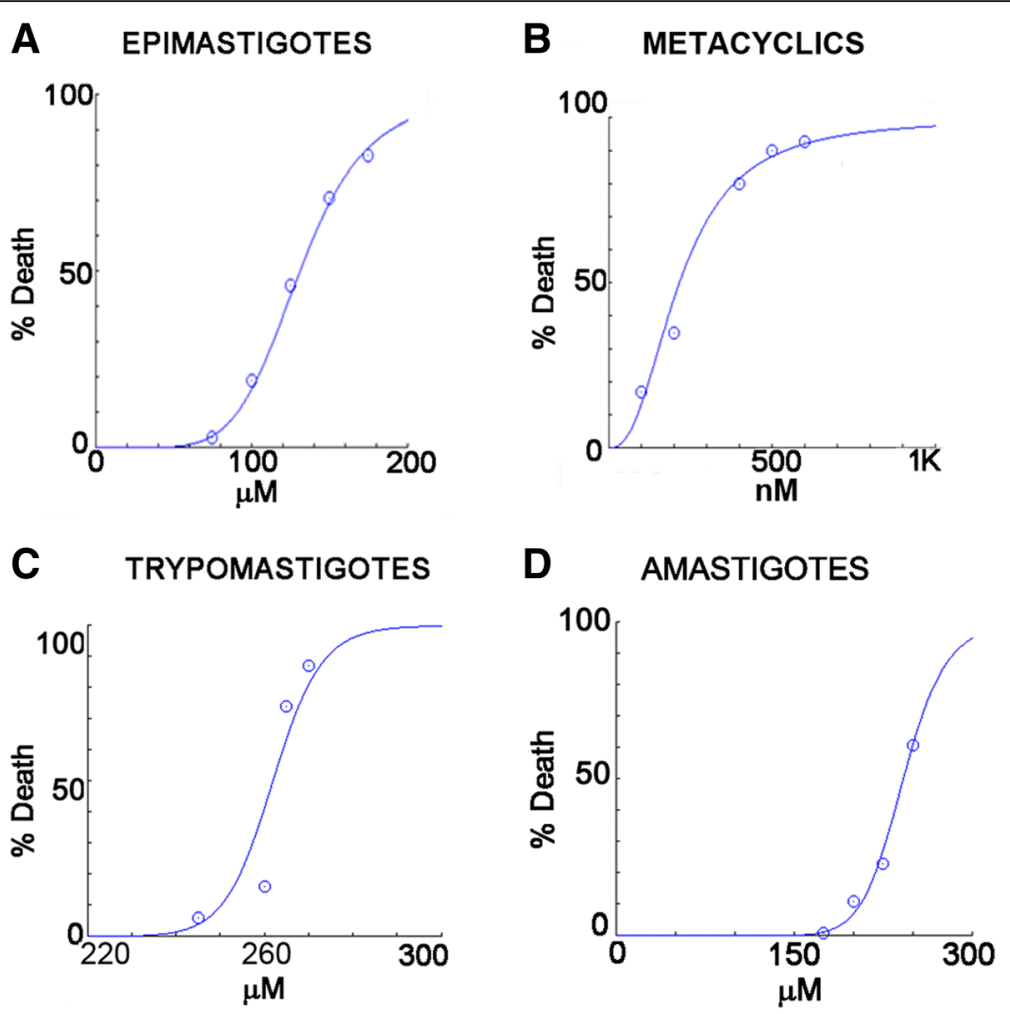

Fig. 1 Effect of 2-BP on different Trypanosoma cruzi developmental forms. a Effect on epimastigotes. $I C_{50}$ value was estimated as $130 \mu M$. $n=3$, $p<0.001$. b Effect on metacyclic trypomastigotes. $I C_{50} / 24$ h value was estimated as $216 \mathrm{nM} . n=3, p<0.001$. $\mathbf{c}$ Effect on culture trypomastigotes. $\mathrm{IC}_{50} / 4 \mathrm{~h}$ value was estimated as $262 \mu \mathrm{M} . n=3, p<0.001$. $\mathbf{d}$ Effect on isolated intracellular amastigotes. $\mathrm{IC}_{50}=242 \mu \mathrm{M} . n=3, p<0.001$

palmitoylated [24, 38]. While untreated epimastigotes (Fig. 2a) and metacyclic trypomastigotes (Fig. 2c), showed prominent flagellar labeling, the 2-BP-treated cells lost their flagellar labeling and showed a disperse reaction throughout the cell body (Fig. $2 \mathrm{~b}$ and $\mathrm{d}$ ).

\section{Morphology, viability and physiology of $T$. cruzi epimastigotes were altered by treatment with $\mathrm{IC}_{50} / 48 \mathrm{~h}$ 2-BP}

2-BP treated epimastigotes had translucent vacuoles at the posterior region and were occasionally linked by the flagella (insets in Fig. 3a and b). Scanning electron microscopy showed a leakage of intracellular material at the flagellar pocket region (Fig 3a and b). Transmission electron microscopy showed large electron lucent vacuoles at the posterior cell region and Golgi alterations (Fig. 3c and d).

To certify that the large vacuoles observed by light and transmission electron microscopy were reservosomes (acidic pre-lysosomal organelles found at the posterior end of T. cruzi epimastigotes), 2-BP-treated epimastigotes were incubated with acridine orange. Analysis by flow cytometry showed a 2-fold increase in the median red fluorescence intensity peak (Additional file 3: Figure S3, left panel). Using fluorescence microscopy, a strong red labeling was found in structures at the posterior region of the treated cells (Additional file 3: Figure S3, right panel), thus indicating that these large vacuoles corresponded to reservosomes.

The size (FSC) and granularity (SSC) of 2-BP-treated epimastigotes were analyzed by flow cytometry, and they showed statistically significant increases of approximately $13 \%$ and $11 \%(p \leq 0.0012$ and $p \leq 0.007)$, respectively, compared to the control (Fig $4 \mathrm{a}$ and b). There was no significant difference in the distribution of cells in the different cell division cycle stages (G1, S and G2-M) between the treated and the control parasites (Fig. 4c).

In mitochondrial viability assays with rhodamine-123, the normalized median of the fluorescence peaks in the stained cells decreased $47 \%(p \leq 0.001)$ in the 2-BP-treated epimastigotes (Fig. 4d), showing that the mitochondrial potential was altered by the palmitoylation inhibition. Only $1.5 \%$ of the population was dead after $48 \mathrm{~h}$ of incubation with 2-BP (Fig. 4e), thus indicating that most parasites were viable.

Endocytosis in T. cruzi epimastigotes is hindered by 2-BP Epimastigotes were treated for $4 \mathrm{~h}$ with $130 \mu \mathrm{M} 2-\mathrm{BP}$, washed and then incubated for $30 \mathrm{~min}$ in LIT medium containing endocytic markers. Two different tracers 


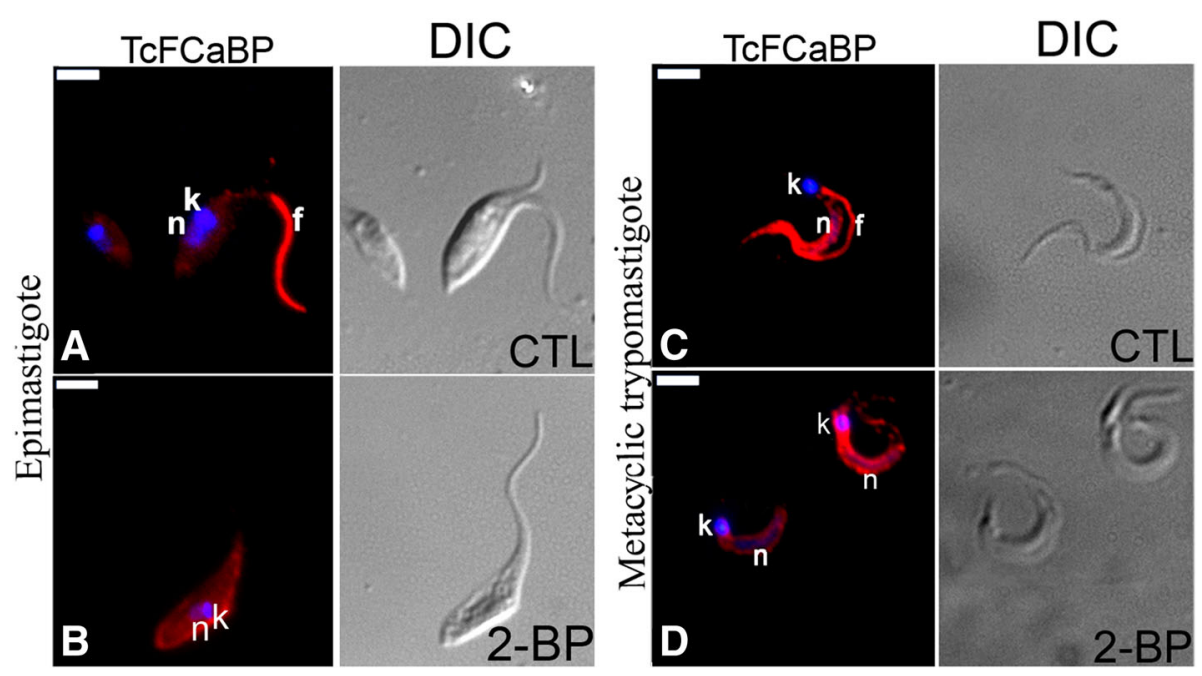

Fig. 2 Localization of TCFCaBP in Trypanosoma cruzi epimastigotes and metacyclic trypomastigotes after 2-BP treatment. a-c Negative control (CTL), showing flagellar localization (in red) of TcFCaBP in epimastigotes (a) and trypomastigotes (c). Nucleus and kinetoplast are stained with DAPI (blue). b-d Incubation with 130 M 2-BP hindered flagellar localization of TcFCaBP. n: nucleus; k: kinetoplast; f: flagellum. Bars $=5 \mu \mathrm{m}$
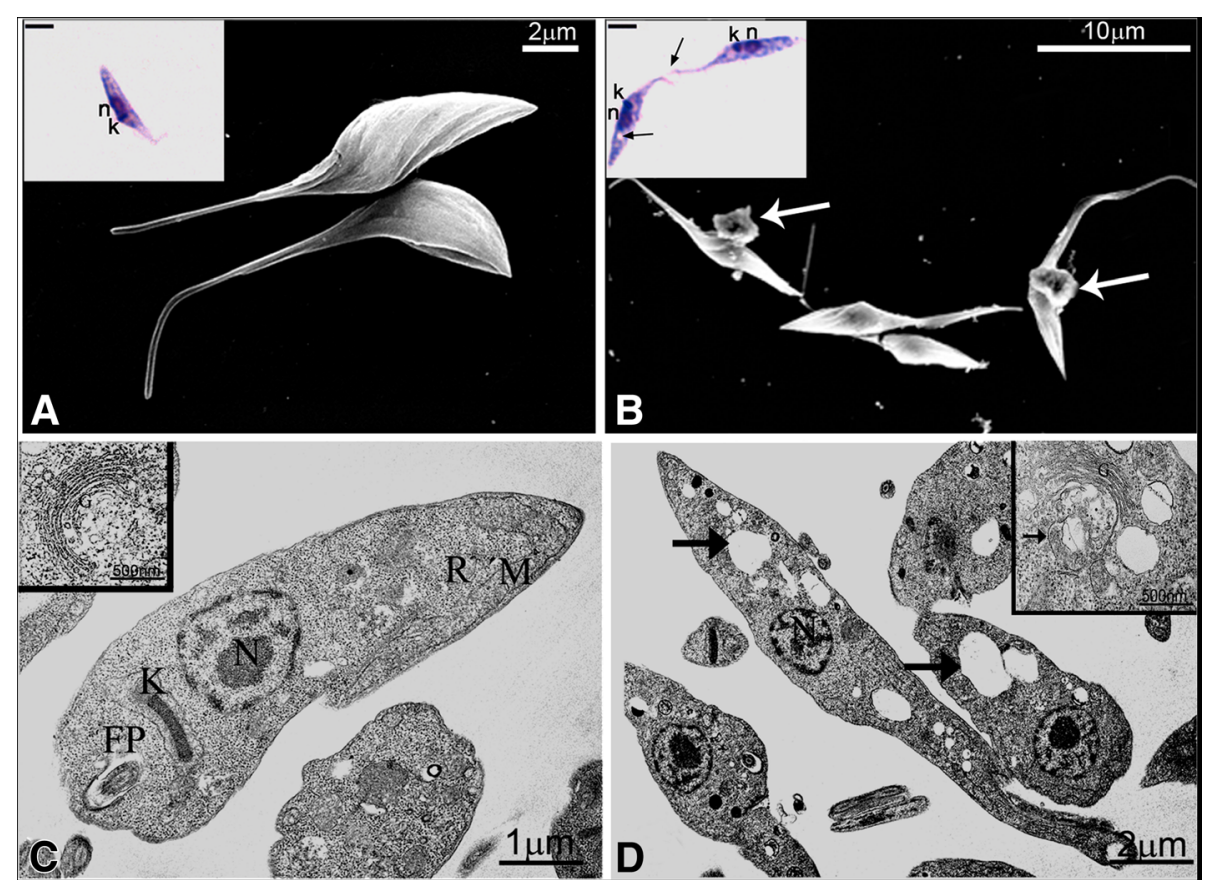

Fig. 3 Morphological alterations of Trypanosoma cruzi epimastigotes treated with $\mathrm{IC}_{50} / 48 \mathrm{~h}$ 2-BP. a-b Scanning electron microscopy of control (a) and 2-BP-treated (b) parasites. Note the extracellular leakage at the flagellar pocket region of treated epimastigotes (write arrows). Insets (bar $=5 \mu \mathrm{m}$ ): bright field microscopy of Giemsa-stained parasites. Control parasite (in a) showing the characteristic elongated shape; treated parasites (in $\mathbf{b}$ ) were larger, with large vacuoles and were adhered by their flagella (black arrows). c-d Transmission electron microscopy. Control epimastigote (c) showing the typical elongated morphology. A representative Golgi complex is shown in the inset. 2-BP-treated parasites (d) presented large electron lucent vacuoles at the anterior tip (black arrows) and Golgi complex alterations (arrow in inset). N: nucleus; K: kinetoplast; FP: flagellar pocket; G: Golgi complex; R: reservosome; M: mitochondrion. Bars $=5 \mu \mathrm{m}$ 

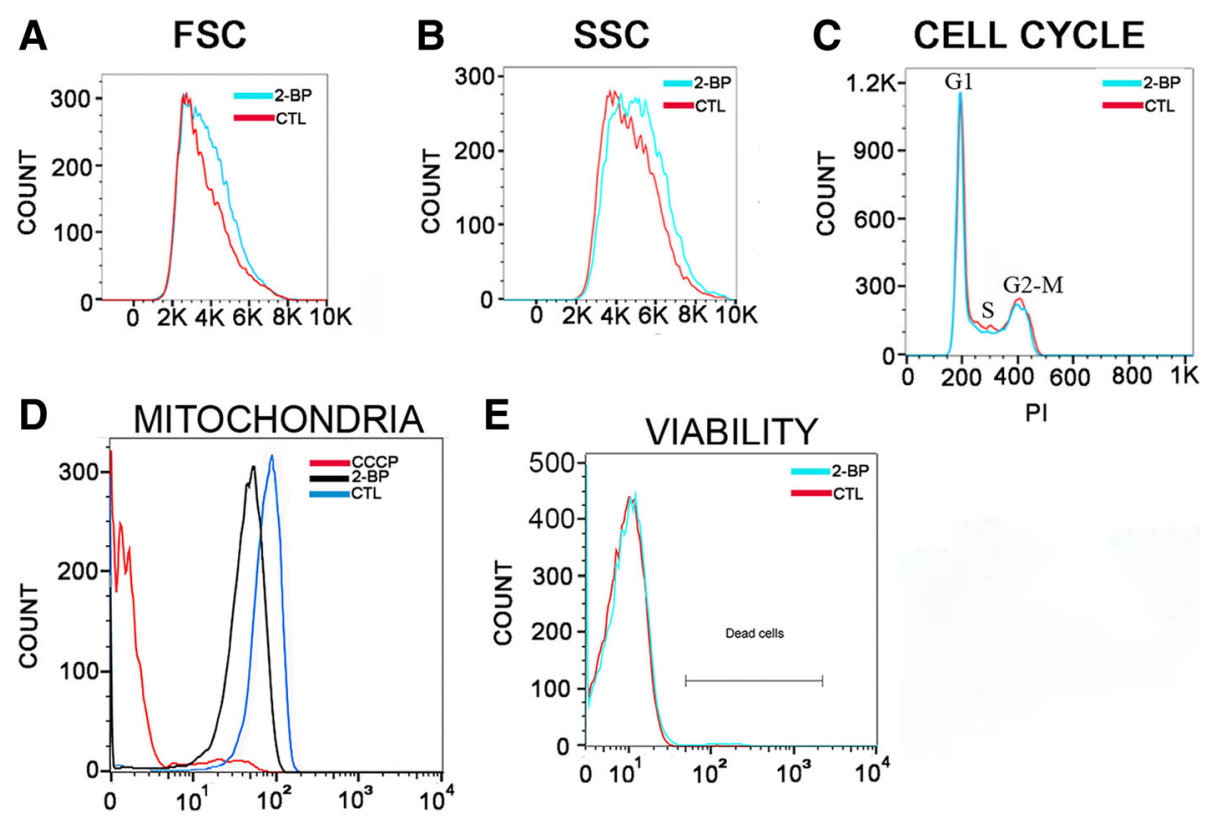

$\mathrm{PI}$

Fig. 4 Effect of incubation with IC50/48 h 2-BP on Trypanosoma cruzi epimastigotes as assessed by flow cytometry. a Forward scatter (FSC) analysis of control (CTL) and treated parasites, showing the larger size of the treated epimastigotes. b Side scatter (SSC) analysis of CTL and 2-BP parasites, showing an increase in granularity. c Cell cycle analysis, showing no difference between CTL and 2-BP treated parasites in the different cell cycle stages. d Mitochondrial potential analysis by rhodamine-123, showing a decrease in the membrane potential for 2-BP-treated epimastigotes; 100 MM CCCP: positive control. e Cell viability analysis of CTL and 2-BP parasites by propidium iodide, showing a few dead cells in 2-BP-treated cultures

were used: transferrin (mostly internalized via the cytostome) and albumin [41].

Incubation with transferrin-Alexa 633 and analysis by flow cytometry showed that 2-BP treated parasites had low endocytic activity, with an approximately $90 \%$ reduction in the normalized 633 fluorescence median (Fig. 5a). Fluorescence microscopy showed that untreated cells internalized transferrin partially in co-localization with the reservosomal marker cruzipain (Fig. 5b). On the other hand, transferrin fluorescence was reduced in 2-BP parasites and no co-localization with cruzipain was observed (Fig. 5c).

Treated epimastigotes incubated with albumin-Alexa 488 showed an approximately $90 \%$ reduction in normalized 488 fluorescence (Fig. 6a). Fluorescence microscopy showed no co-localization with cruzipain and little albumin was ingested (Fig. $6 \mathrm{~b}$ and d).

\section{Trypanosoma cruzi metacyclogenesis is altered by 2-BP}

The metacyclic trypomastigotes/epimastigotes ratio was evaluated after $72 \mathrm{~h}$ in the supernatant of the TAU3AAG differentiation medium (Fig. 7a). While in the control this ratio was approximately 5:1, for 2-BP-treated parasites the ratio ranged from $0.5: 1$ (2-BP in pre-stress medium) to 2.5:1 (2-BP in post-stress medium) (Fig. 7b). Incubation with TcFCaBP $\mathrm{mAb}$ showed that all treatments (2-BP in pre-stress, stress or post-stress media) led to mislocalization of this flagellar protein, which was found at the parasite surface, but not at the flagellum.
Morphology of parasites from the culture supernatants was analyzed by light microscopy. Metacyclic trypomastigotes were abundant in the untreated control (Fig. 7c). When 2-BP was added to pre-stress assays, then epimastigotes prevailed (Fig. 7c) and the few detected metacyclic trypomastigotes were smaller, with their nucleus and kinetoplast closely located. When 2-BP was added to the stress and post-stress assays, round cells prevailed and the few detected metacyclic trypomastigotes were morphologically similar to those of the control (Fig. 7c).

\section{Infectivity of $T$. cruzi trypomastigotes is altered by 2-BP}

Trypomastigotes obtained from the TAU3AAG supernatants of the 2-BP metacyclogenesis assay were used to infect Vero cells. Six days after infection, the released trypomastigotes were counted and the parasite number was compared to that of trypomastigotes collected from Vero cell cultures that were infected with untreated parasites. There was a significant inhibition in the number of released trypomastigotes for all 2-BP treatments, ranging from 48.5 to $75 \%$ (Fig. 8a).

Light microscopy showed round-shaped, smaller and thicker parasites with a round nucleus in all treated groups when compared to the control trypomastigotes (Fig. 8a). Released trypomastigotes from the control and pre-stress assays were incubated with TcFCaBP $\mathrm{mAb}$ and were visualized by fluorescence microscopy (Fig. 8b). In the control parasites protein localization was enriched in 
A
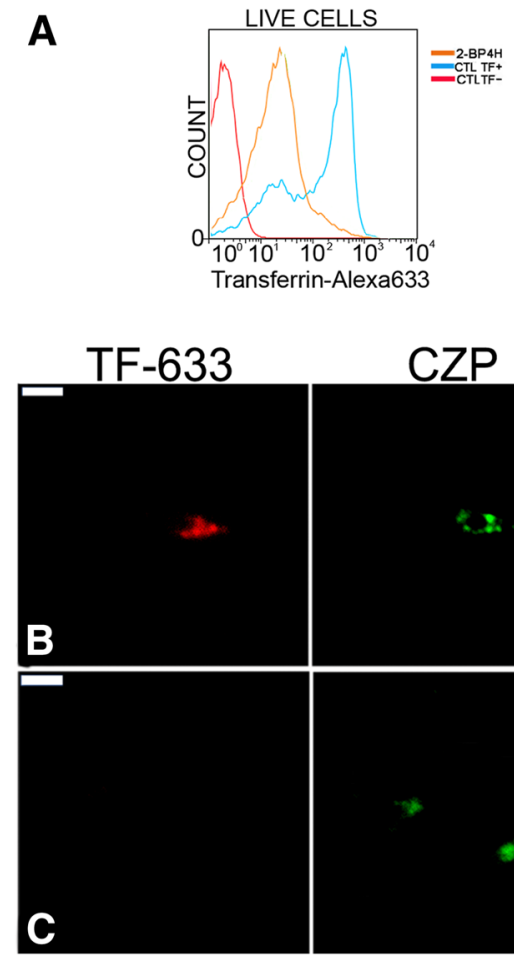
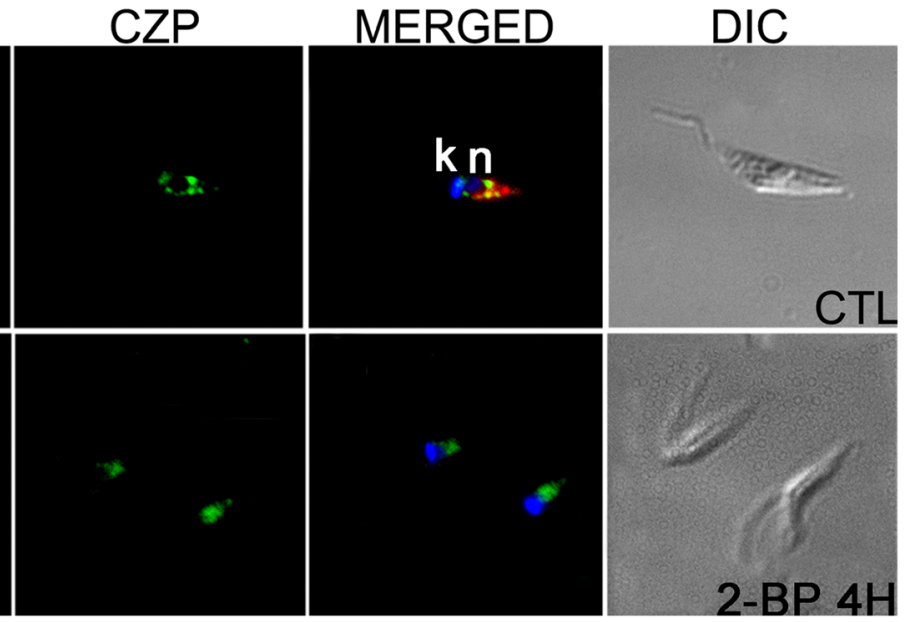

Fig. 5 Transferrin-AlexaFluor 633 endocytosis is altered in Trypanosoma cruzi epimastigotes by 2-BP treatment. a Flow cytometry analysis showing that transferrin internalization was inhibited after $4 \mathrm{~h}$ of treatment with 2-BP. ${ }^{* * *}: p<0.001$. b Control cell: co-localization of internalized transferrin (TF-633) with cruzipain (CZP) in reservosomes by fluorescence microscopy. c Parasites treated for $4 \mathrm{~h}$ with 2-BP showing no fluorescence signal for transferrin. $\mathrm{n}$ : nucleus; $\mathrm{k}$ : kinetoplast. Bars $=5 \mu \mathrm{m}$

their flagella (Fig. 8b), while in the trypomastigotes from the 2-BP experiments the localization was diffuse on the cell surface or was partially located at the flagellar membrane (Fig. 8b).

In another experiment, cell-derived trypomastigotes were treated with $\mathrm{IC}_{50} 2-\mathrm{BP}(262 \mu \mathrm{M})$ and then used to infect Vero cells. At this experimental point (before infection), aliquots of the treated and untreated (control) trypomastigotes were incubated with the TcFCaBP mAb. In untreated trypomastigotes the labeling was in the flagellum, while in treated parasites the labeling was diffuse in the cytoplasm, with a few trypomastigotes presenting flagellar labeling (Fig. 9a, "before infection"). After four days of infection the released trypomastigotes were collected and incubated with the TcFCaBP mAb. The same result was obtained: while in trypomastigotes from the control experiment the labeling was in the flagellum (Fig. 9a, "after infection"), in trypomastigotes obtained from the 2-BP treatment assay the labeling was diffuse in the cytoplasm, with a few trypomastigotes presenting flagellar labeling (Fig. 9a, "after infection"). The released trypomastigotes were counted and their numbers were compared. There was a $45.5 \%$ inhibition in the number of released parasites in the treatment assay (Fig. 9b). The morphology of the released parasites from the treatment assay was analyzed by light microscopy, showing round-shaped, smaller, thicker cells with a rounded nucleus, when compared to the control trypomastigotes (Fig. 9c).

\section{Effect of palmitate on different $T$. cruzi developmental forms}

Epimastigotes treated with $130 \mu \mathrm{M}\left(\mathrm{IC}_{50} / 48 \mathrm{~h}\right.$ value $)$ palmitate showed no significant alteration in growth, the parasite number decreasing only in $13.4 \%$ when compared to untreated cells (Fig. 10a, epimastigotes). Metacyclic trypomastigotes remained alive after incubation with $216 \mathrm{nM}\left(\mathrm{IC}_{50} / 24 \mathrm{~h}\right.$ value), the parasite number decreasing only in $8.5 \%$ when compared to untreated cells (Fig. 10a, metacyclics). Treatment of cell-derived trypomastigotes for $4 \mathrm{~h}$ with $262 \mu \mathrm{M}\left(\mathrm{IC}_{50} / 4 \mathrm{~h}\right.$ value) palmitate had low effect on infectivity, with decrease of $19.5 \%$ in the number of released trypomastigotes (Fig. 10a, trypomastigotes). Furthermore, TcFCaBP subcellular localization was not altered in all these developmental forms (Fig. 10b).

Epimastigotes treated with $130 \mu \mathrm{M}\left(\mathrm{IC}_{50} / 48 \mathrm{~h}\right.$ value $)$ palmitate showed a reduction in $27.5 \%$ of the mitochondrial potential after rhodamine 123 incubation (Additional file 4: Figure S4), as compared to $47 \%$ reduction in 2 -BP treated cells. 
A
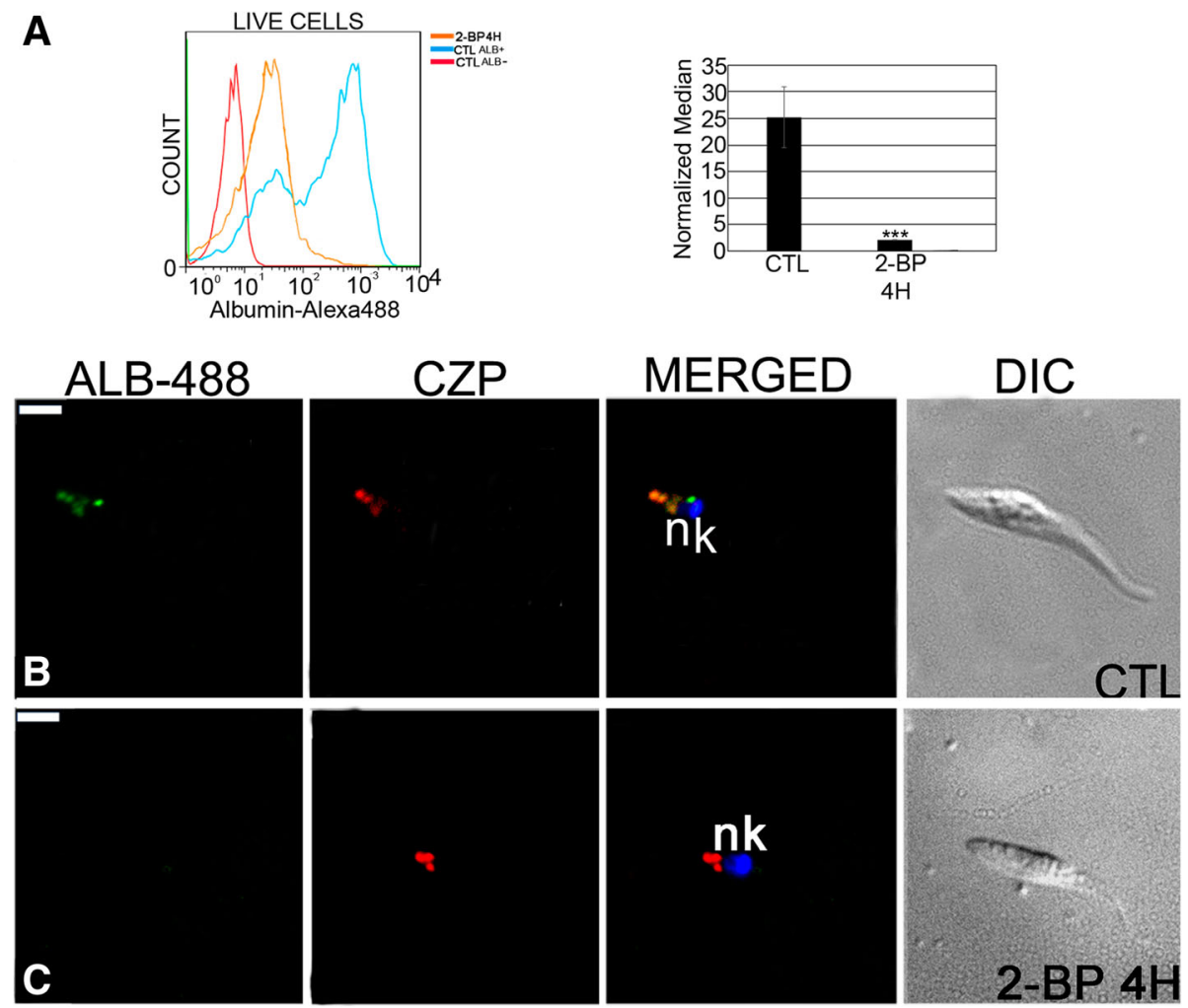

Fig. 6 Albumin-Alexa 488 endocytosis is altered in Trypanosoma cruzi epimastigotes by 2-BP treatment. a Flow cytometry analysis showing that albumin internalization was inhibited after treatment for $4 \mathrm{~h}$ with 2-BP. ${ }^{* * *}: p<0.001$. b Control: Co-localization of internalized albumin (ALB-488) with cruzipain (CZP) in reservosomes by fluorescence microscopy. c Parasites treated for $4 \mathrm{~h}$ with 2-BP showing no fluorescence signal of albumin (no co-localization with cruzipain). N: nucleus; k: kinetoplast. Bars $=5 \mu \mathrm{m}$

\section{Discussion}

Protein palmitoylation promotes membrane localization, regulation of enzymatic activity, regulation of gene expression and protein stability [1-3]. Many palmitoylated proteins are important for diverse aspects of pathogenesis in eukaryotic parasites, including differentiation into infective life cycle stages, biogenesis and tethering of secretory organelles, assembling the machinery powering motility and targeting virulence factors to the plasma membrane [42]. Here we analyzed the effect 2-BP, a palmitate analogue that can inhibit palmitoylation, on the different life stages of the pathogenic protozoan T. cruzi.

T. cruzi has two main developmental forms when in the vertebrate host: intracellular amastigotes and bloodstream trypomastigotes. Assays on amastigotes are relevant as amastigotes are responsible for tissue damage and trypomastigote formation [43]. The existence of an intracellular epimastigote-like form as an intermediate stage within the mammalian host, morphologically and biochemically similar to the non-infectious extracellular epimastigote form [44], supports the preliminary screening of compounds on the non-infectious stage of the parasite [45]. Therefore, data concerning all $T$. cruzi developmental forms (epimastigotes, amastigotes and trypomastigotes) are crucial to explore the basic cell biology of Trypanosoma cruzi.

The repertoire of T. cruzi palmitoyl transferases (PATs) was first checked and 15 putative proteins were found [27], which agrees with Goldston and coworkers [28]. All respective genes, except for TcPAT6, were successfully isolated by PCR, thus indicating that $T$. cruzi has the palmitoylation machinery in its genome. Indeed, dynamic S-palmitoylation machinery could be expressed in T. cruzi epimastigotes [27]. Therefore, 2-BP could be exploited to gain some information on this lipid modification in this parasite, since it has been already demonstrated that 2-BP inhibits some PATs in T. brucei [19].

T. cruzi culture epimastigotes incubated with $130 \mu \mathrm{M}$ 2-BP $\left(\mathrm{IC}_{50} / 48 \mathrm{~h}\right)$ did not show flagellar localization of TcFCaBP (a flagellar protein that is known to be palmitoylated), which agrees with the data obtained with $T$. cruzi TcFCaBP palmitoylation-deficient mutants (C4A and $\Delta \mathrm{N})$ [24], thus indicating that 2-BP also inhibits palmitoylation in T. cruzi epimastigotes. TcFCaBP was also mislocated in metacyclic and culture trypomastigotes that were incubated with an $\mathrm{IC}_{50}$ value of 2-BP (216 nM and $262 \mu \mathrm{M}$, respectively), thus suggesting that palmitoylation could be important modification for protein 


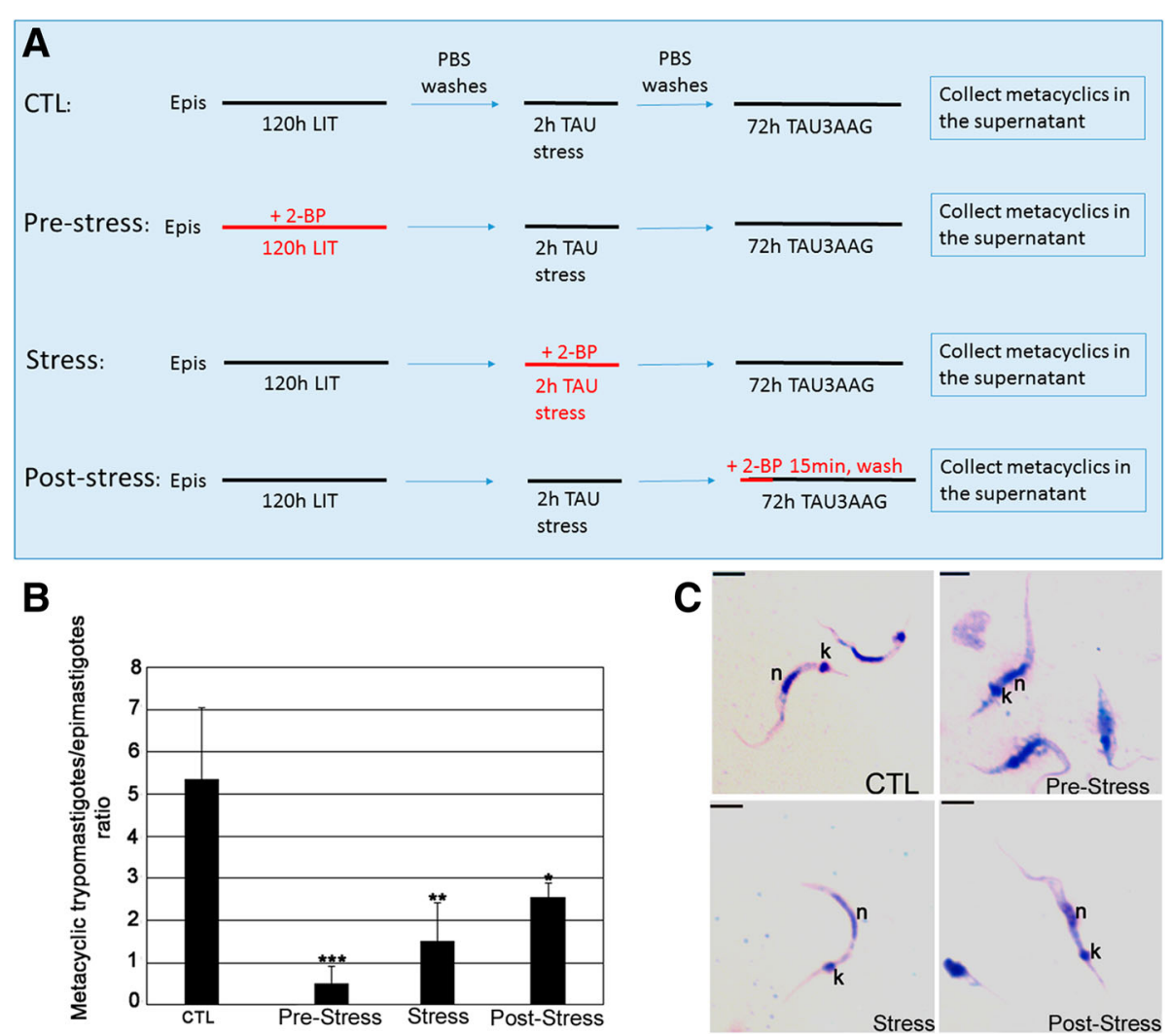

Fig. 7 Metacyclogenesis of Trypanosoma cruzi is inhibited by 2-BP treatment. a Schematic view of the metacyclogenesis experimental design. $\mathbf{b}$ Ratio of metacyclic trypomastigotes/epimastigotes after $72 \mathrm{~h}$ in the supernatant of TAU3AAG medium; the ratio decreased in all treatments, when compared to the untreated control. *: $p>0.005 ; *^{* *} p<0.005 ;{ }^{* * *}: p<0.001$. c Giemsa-stained cells collected in the supernatant showing morphological alterations after the different treatments. $\mathrm{n}$ : nucleus; $\mathrm{k}$ : kinetoplast. Bars $=5 \mu \mathrm{m}$

localization in T. cruzi. While the data points to a new pharmacological sensitivity to 2-BP in the metacyclic stage, this could just as likely be a dependence on fatty acid synthesis, or any number of other cellular enzymes that are inhibited by micromolar concentrations of 2-BP, as it has been shown that 2-BP is broadly reactive across hundreds of cellular proteins at low micromolar concentrations in mammalian cells [21]. On the other hand, trypanosomatids are unicellular organism with a large evolutionary distance to mammalian cells [46, 47]. The earliest forms of T. cruzi itself are deduced to have been associated with marsupial opossums at the time of separation of South America from Gondwanaland about 40 million years ago [48]. Consequently, it is also possible that 2-BP is not so broadly reactive in $T$. cruzi due to the parasite own protein repertoire. Further studies are needed to clarify this point.

Interestingly, incubation with palmitate did not alter epimastigote growth, metacyclic trypomastigote viability and TcFCaBP flagellar localization in the different developmental forms, thus indicating that the alterations observed in our study were due to the 2-BP treatment. However, it is still unclear if 2-BP operates by targeting a specific mechanism, as it has been shown that 2-BP is a promiscuous inhibitor of membrane-bound enzymes [21, 49]. Further experiments are needed to elucidate this point.

Incubation of infected Vero cells for $72 \mathrm{~h}$ with 2-BP resulted in a $40 \%$ increase in the number of released trypomastigotes. It is possible that 2-BP treatment led to deregulation of metabolic pathways (e.g., energy production, nucleotide metabolism, pteridine biosynthesis and/ or fatty acid oxidation) in the host cells or in the intracellular parasites, which are key processes for the parasite intracellular development [50], accelerating the parasite intracellular differentiation cycle. Indeed, several enzymes and transporters for the above mentioned metabolic pathways were already identified in the T. brucei palmitoylome [19].

2-BP-treated epimastigotes showed marked morphological alterations. The large vesicles close to the Golgi complex that were observed by transmission electron microscopy could be a result of inhibition of palmitoylation, leading to accumulation of depalmitoylated proteins in vesicles at the trans-Golgi network, since it is known that the Golgi compartment acts as a hub for 

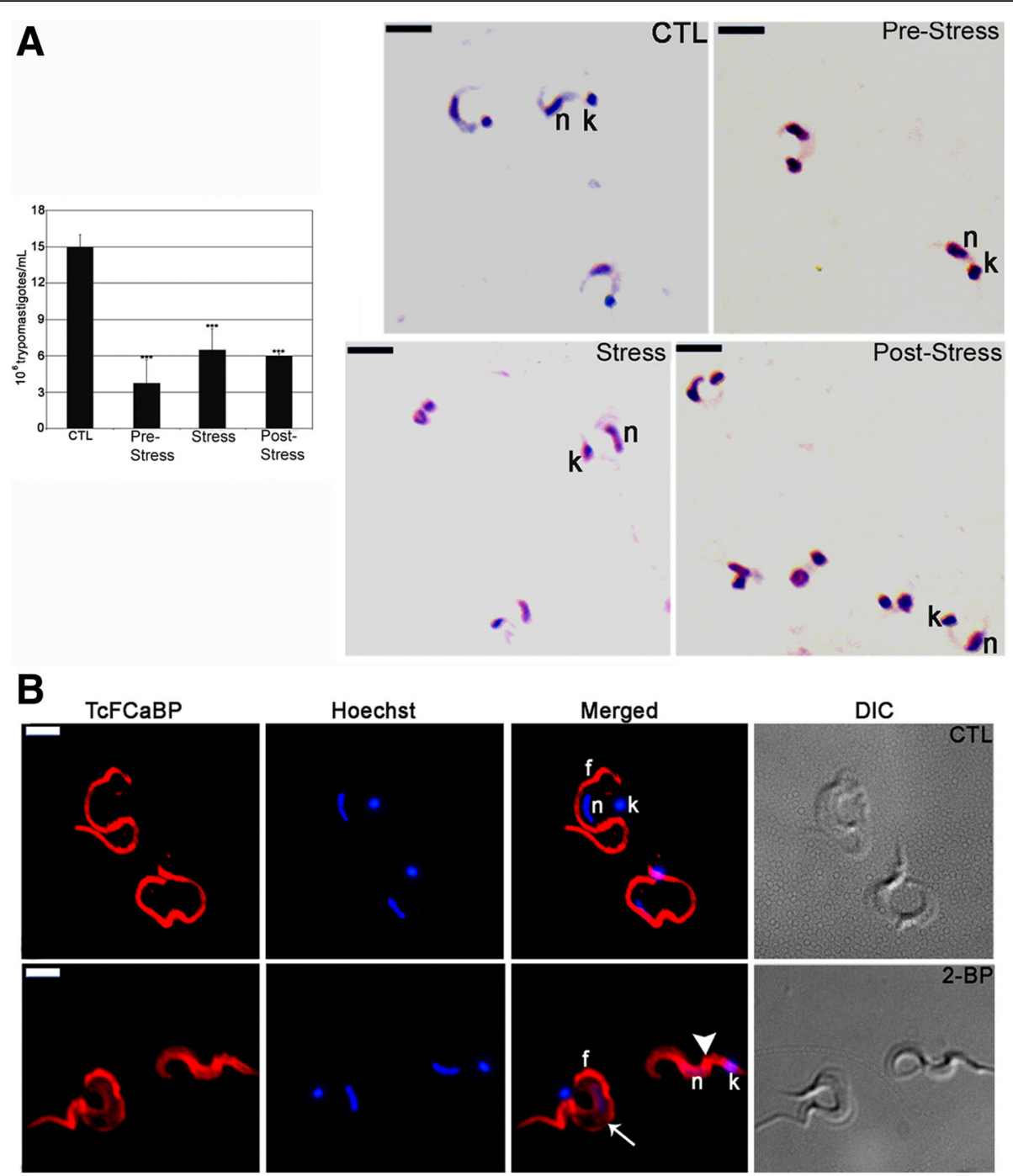

Fig. 8 2-BP treatment during metacyclogenesis alters Trypanosoma cruzi host cell infectivity. a Number of released cell-culture trypomastigotes (CTL and 2-BP-treated groups) after Vero cell infection, showing a reduction of approximately $45.5 \%$ to $75 \%$ for the treated groups when compared to the control parasites. $n=3,{ }^{* * *}: p<0.001$. Released cell-culture trypomastigotes (CTL and 2-BP-treated groups) as visualized by light microscopy showing morphological alterations, such as smaller size and round nucleus, compared to the control parasites. n: nucleus; k: kinetoplast. Bars $=5 \mu \mathrm{m}$. $\mathbf{b}$ Localization of TcFCaBP of Trypanosoma cruzi trypomastigotes released from Vero cells infected with 2-BP-treated metacyclic trypomastigotes (pre-stress group). CTL: Negative control showing a strong labeling in the flagellum. 2-BP: Treated parasites showing cellular (arrowhead) and partial flagellar (arrow) labeling. n: nucleus; k: kinetoplast; f: flagellum. Bars $=5 \mu \mathrm{m}$

palmitoylation [51]. Moreover, mostly overexpressed FLAG-tagged TcPATs were found as single spots at the parasite anterior end, which could be the Golgi complex [27]. It is possible that the vesicles observed close to the Golgi could leave the flagellar pocket by exocytosis, thus forming the extracellular material observed by scanning electron microscopy.

2-BP-treated epimastigotes showed a reduction of $47 \%$ in mitochondrial membrane potential, as opposed to $27.5 \%$ in palmitate-treated parasites, thus indicating that the membrane potential alteration was more likely due to the 2-BP effect than to a lipidic stress. It has been recently shown that an active and dynamic S-depalmitoylation is present in mitochondria, regulating S-palmitoylation [52]. It is thus tempting to speculate that S-palmitoylation also occurs in the T. cruzi mitochondrion.

Internalization of transferrin and albumin was inhibited in the 2-BP-treated epimastigotes. Our data on transferrin inhibition agree with a former work on the inhibition of diferric transferrin receptor-mediated endocytosis that is associated with palmitoylation of the transferrin receptor [53], thus suggesting that the transferrin receptor of T. cruzi could be palmitoylated. However, the existence of a transferrin receptor in $T$. cruzi has been proposed [54], but this receptor has been not yet identified. Our data indicate that the two endocytic 

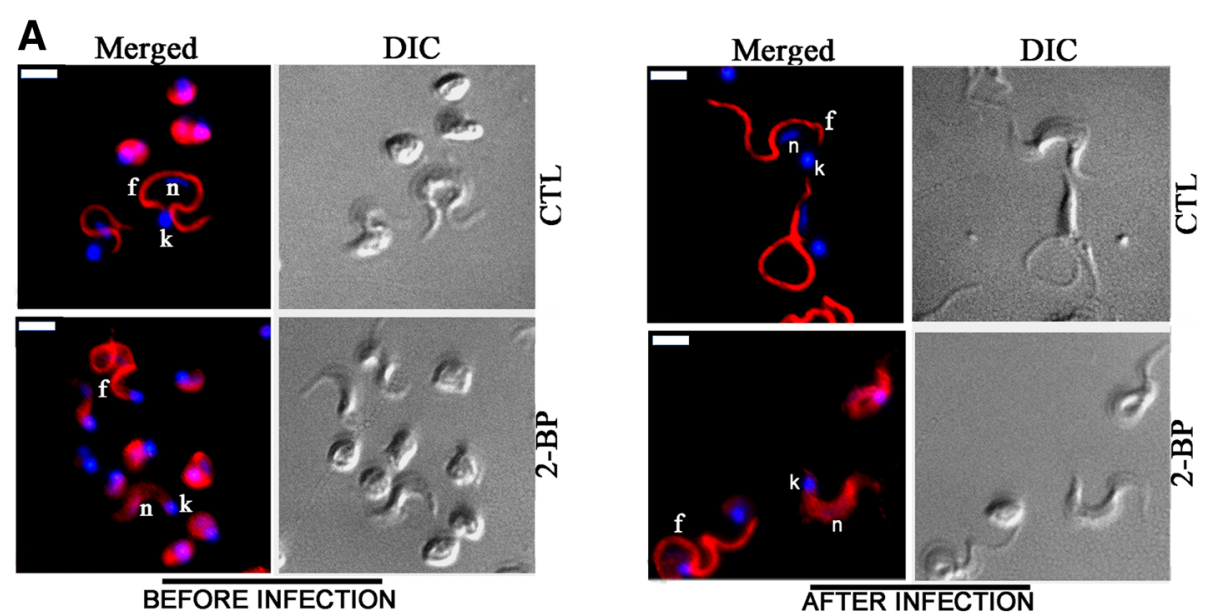

B

C
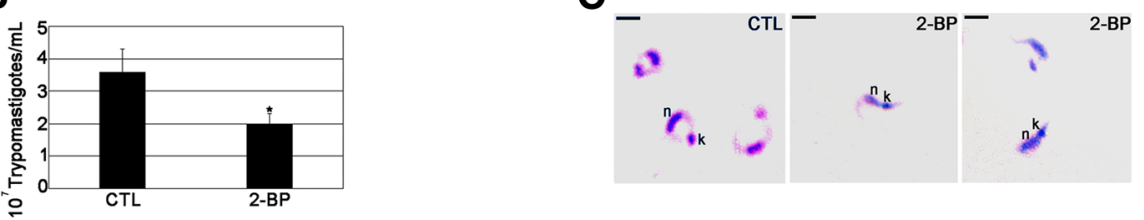

Fig. 9 2-BP treatment of culture trypomastigotes with $I_{50}$ 2-BP alters Trypanosoma cruzi host cell infectivity. a 2-BP treatment before infection altered TCFCaBP flagellar localization in parasites obtained before and after infection. $\mathbf{b}$ Note the decrease in number of released trypomastigotes four days after infection. *: $p>0.05$ (c) Giemsa staining of released trypomastigotes showing morphological alterations. n: nucleus; k:

kinetoplast. Bars $=5 \mu \mathrm{m}$

portals of T. cruzi epimastigotes, the cytostome and the flagellar pocket $[38,55]$, are deficient for transferrin/albumin internalization when the parasites were treated. Accordingly, the pellet of 2-BP-treated epimastigotes was paler than that of the control parasites (data not shown), indicating that treated parasites were deficient in incorporating hemin from the LIT medium.

The translucent vacuoles observed by light microscopy at the posterior end of epimastigotes corresponded to the large, electron-lucent vacuoles found by transmission electron microscopy. Incubation with acridine orange showed an approximately 99\% increase of the fluorescence signal by flow cytometry and a stronger red labeling by fluorescence microscopy at the posterior cell end, indicating that these large vacuoles correspond to the reservosomes, acidic organelles that accumulate ingested proteins [55, 56]. The lower endocytic capacity of 2-BP-treated epimastigotes resulted in the appearance of these less dense reservosomes.

It has been proposed that the content of reservosomes is metabolized during the metacyclogenesis process [56]. Considering that the 2-BP-treated epimastigotes had poor endocytic activity, we analyzed the effect of incubating epimastigotes with 2-BP before metacyclogenesis (pre-stress assay). As a result, treated epimastigotes had a decreased ability to differentiate (up to $75 \%$ ). Light microscopy showed that some resulting metacyclic forms had a nucleus close to the kinetoplast, indicating that the development of the differentiation process was affected. Therefore, it seems that epimastigotes bearing reservosomes with low protein content have decreased aptitude for the differentiation process.

All morphological alterations found in 2-BP stressed epimastigotes indicate that the treatment was highly detrimental for differentiation and infectivity. When the resulting metacyclic trypomastigote forms were submitted to an infection assay, the percentage of released trypomastigotes decreased from 48.5 to $25 \%$, demonstrating that the treatment interfered with parasite infectivity, possibly due to loss of surface proteins involved in host cell interactions. Metacyclic trypomastigotes obtained from the pre-stress metacyclogenesis assays were used to infect Vero cell cultures. As a result, released trypomastigotes (i.e., after 10 days without treatment with 2-BP) still showed TcFCaBP mislocalization and nuclear/kinetoplast morphological alterations, which could contribute to reduce the number of released parasites.

We submitted cell-derived trypomastigotes treated with $\mathrm{IC}_{50} 2$-BP to a host cell interaction assay to determine whether the treatment could result in reduced infectivity. The number of released trypomastigotes decreased $45.5 \%$ in the treated group, demonstrating that the treatment interfered with host cell interactions. Compared to the control trypomastigotes, the treated parasites had round-shaped, smaller, thicker cell bodies with a round nucleus, together with mislocalization of 

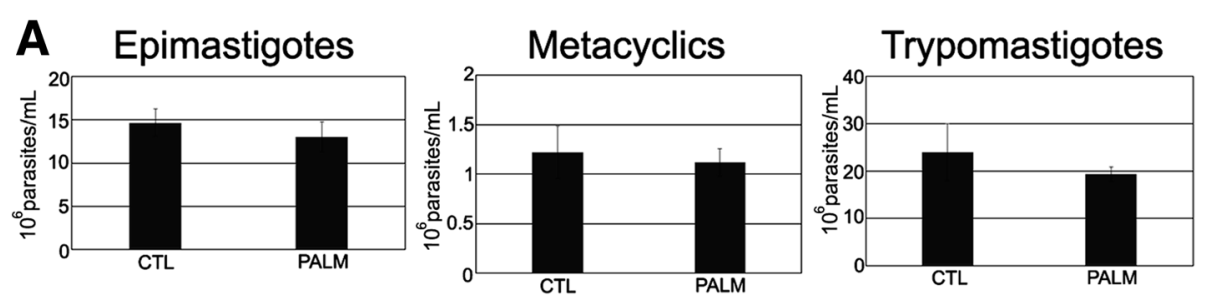

B

Epimastigotes
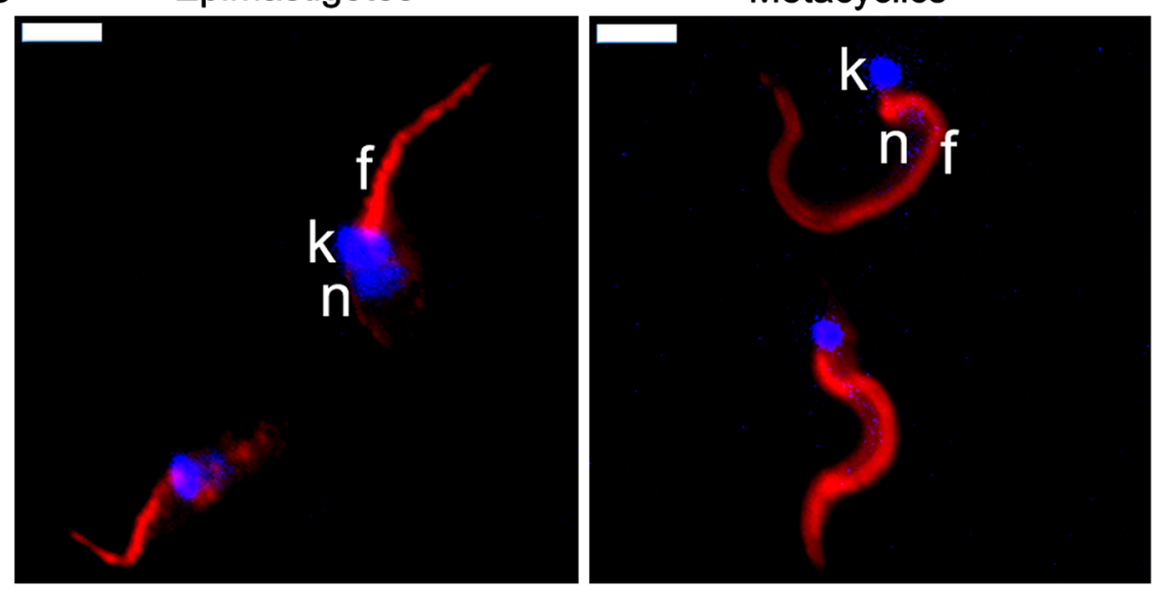

Trypomastigotes

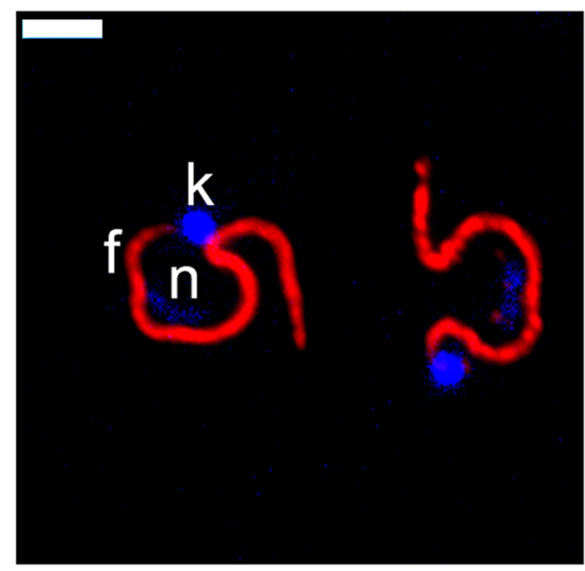

Before infection

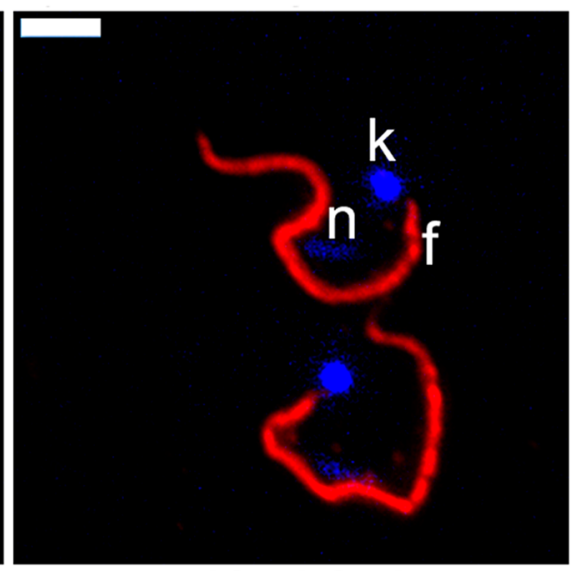

After infection

Fig. 10 Effect of palmitate on Trypanosoma cruzi. Different developmental forms were incubated with palmitate, using the respective $I C_{50}$ values of 2-BP. a No effect was observed on epimastigotes growth, metacyclics viability and cell-derived trypomastigotes infectivity. $\mathbf{b}$ Localization of TCFCaBP was not altered in all developmental forms tested. n: nucleus; k: kinetoplast; f: flagellum. Bars $=5 \mu \mathrm{m}$

TcFCaBP. On the other hand, low reduction in infectivity and no TcFCaBP mislocalization were found in palmitate treated parasites. These results suggest that palmitoylation was altered in 2-BP treated trypomastigotes.

Our data indicate that 2-BP affects the morphology, endocytosis, differentiation and infectivity of T. cruzi, thus suggesting that these functions could be somehow linked to protein palmitoylation. Our major finding on metacyclic trypomastigotes suggests a unique target dependency during T. cruzi development that is suitable for pharmacological rationales. The next step is to identify and validate the biochemical pathways involved with palmitoylation that lead to these alterations by proteomic and reverse genetic approaches. Future studies focusing on the characterization of these pathways are also paramount to understand the role of palmitoylation-dependent protein localization in parasite survival.

Click-enabled activity-based probes have been used for profiling the targets of 2-BP inhibition. It has been shown that the probes preferentially labeled the active site of DHHC PATs, but similarly labeled hundreds of other proteins, including transporters, channels, enzymes, 
and chaperones [21]. Therefore, it is recognized that 2-BP is more likely a non-selective membrane tethered cysteine alkylator with many targets beyond palmitoyl transferases $[21,49]$. Thus, although data on the sensitivity of T. cruzi for 2-BP cannot be linked solely to palmitoylation, we cannot rule out the possibility that palmitoylation is involved in some or all events here reported (due to the TcFCaBP mislocalization). The challenging search of specific palmitoylation inhibitors is a crucial step to determine the role of palmitoylation on the T. cruzi biology.

Some palmitoylation inhibitor compounds have been already described [57], and one of them - Compound V (CV) - behaved similarly to 2-BP, in that it inhibited all four of the DHHC proteins tested. 2-BP and CV inhibited autoacylation of the PAT enzyme, which is tightly correlated with the ability to transfer palmitate to substrate [58]. Future works with other specific palmitoylation inhibitors against $T$. cruzi could elucidate the role played by palmitoylation in the parasite life cycle and could lead to a potential inhibitor of parasite infection with validated targets.

\section{Conclusions}

2-bromopalmitate treatment of Trypanosoma cruzi altered parasite morphology, endocytosis, differentiation and infectivity, indicating that 2-BP inhibits key cellular processes of $T$. cruzi that may be regulated by palmitoylation of vital proteins. Palmitoylation is an important cellular process that may be a good target for further cellular/molecular biology studies with specific palmitoylation inhibitors in order to elucidate the life cycle of $T$. cruzi. Our major finding on metacyclic trypomastigotes suggests a unique target dependency during $T$. cruzi development that is suitable for pharmacological rationales.

\section{Additional files}

\section{Additional file 1: Figure S1. Trypanosoma cruzi PATs genes}

amplification by PCR as analyzed by $1 \%$ agarose gel. Note the expected amplifications for all PATs genes, except TCPAT6. $\mathrm{Kb}=1 \mathrm{~Kb}$ plus ladder. (TIF $141 \mathrm{~kb}$ )

Additional file 2: Figure S2. 48 h-old Trypanosoma cruzi intracellular parasites isolated by cavitation after $24 \mathrm{~h}$ with $125 \mu \mathrm{M}$ 2-BP. A) Control isolated amastigote incubated in DMEM medium with $0.125 \%$ DMSO; B) Treated parasite with an intermediate trypomastigote-like morphology, with kinetoplast close to the nucleus; C) Treated parasite showing the typical trypomastigote form. $n=$ nucleus; $k=$ kinetoplast. Bars $=5 \mu \mathrm{m}$. (TIF $332 \mathrm{~kb}$ )

Additional file 3: Figure S3. Characterization of acid compartments in control (CTL) and 2-BP-treated epimastigotes after acridine orange (AO) staining. Note the increased AO fluorescence signal in 2-BP parasites (left panel), which corresponds to increased fluorescence in large vacuoles at the posterior cell end (right panel). Bars $=5 \mu \mathrm{m}$. (TIF $279 \mathrm{~kb}$ )

Additional file 4: Figure S4. Effect of incubation with $130 \mu \mathrm{M}$ palmitate on mitochondrial potential of Trypanosoma cruzi epimastigotes. Analysis by flow cytometry using rhodamine- 123 shows a decrease of $27.5 \%$ in the mitochondrial membrane potential in palmitate-treated cells. $100 \mu \mathrm{M}$ CCCP: positive control. (TIF 99 kb)

\section{Acknowledgements}

The authors thank the Program for Technological Development in Tools for Health-PDTIS-FIOCRUZ for use of its facilities (Flow Cytometry Platform RPT08L and Confocal and Electronic Microscopy Platform RPT07C at the Instituto Carlos Chagas/Fiocruz-PR, Brazil). The authors also thank Dr. Robert Brown for critically reading this manuscript and Vanessa Martins for her technical support on parasite cultures.

\section{Funding}

This work was supported by CNPq, CAPES and Fiocruz.

\section{Availability of data and materials}

All data generated or analyzed during this study are included in this published article and its Additional files.

\section{Authors' contributions}

CMB planned and performed the experiments, analyzed the data and wrote the manuscript. RLK acquired, analyzed the flow cytometry data and help to write the paper. IE also planned experiments, analyzed the data and revised the manuscript. MJS conceived the study and edited the final version of the manuscript. All authors read and approved the final manuscript.

Ethics approval and consent to participate

Not applicable.

\section{Consent for publication}

Not applicable.

\section{Competing interests}

The authors declare that they have no competing interests.

\section{Publisher's Note}

Springer Nature remains neutral with regard to jurisdictional claims in published maps and institutional affiliations.

\section{Author details}

${ }^{1}$ Laboratory of Cell Biology, Carlos Chagas Institute/Fiocruz-PR, 81310-020 Curitiba, Paraná, Brazil. ${ }^{2}$ Laboratory of Functional Genomics, Carlos Chagas Institute/Fiocruz-PR, 81310-020 Curitiba, Paraná, Brazil. ${ }^{3}$ Mammalian Cell Biotechnology Laboratory, Molecular Biology Institute of Paraná (IBMP), 81310-020 Curitiba, Paraná, Brazil. ${ }^{4}$ Department of General Biology, State University of Ponta Grossa, 84010-290 Ponta Grossa, Paraná, Brazil.

Received: 12 January 2018 Accepted: 27 August 2018

Published online: 31 August 2018

\section{References}

1. Fukata Y, Murakami T, Yokoi N, Fukata M. Local palmitoylation cycles and specialized membrane domain organization. Curr Top Membr. 2016;77:97-141.

2. Blaskovic S, Adibekian A, Blanc M, van der Goot GF. Mechanistic effects of protein palmitoylation and the cellular consequences thereof. Chem Phys Lipids. 2014;180:44-52.

3. Corvi MM, Berthiaume LG, De Napoli MG. Protein palmitoylation in protozoan parasites. Front Biosci. 2011;3:1067-79.

4. Conibear E, Davis NG. Palmitoylation and depalmitoylation dynamics at a glance. J Cell Sci. 2010;123:4007-10.

5. Martin BR, Cravatt BF. Large-scale profiling of palmitoylation in mammalian cells. Nat Methods. 2009;6:135-8.

6. Sanders SS, Martin DD, Butland SL, Lavallée-Adam M, Calzolari D, Kay C, et al. Curation of the mammalian palmitoylome indicates a pivotal role for palmitoylation in diseases and disorders of the nervous system and cancers. PLoS Comput Biol. 2015;11:e1004405.

7. Martin BR, Wang C, Adibekian A, Tully SE, Cravatt BF. Global profiling of dynamic protein palmitoylation. Nat Methods. 2012;9:84-9.

8. Roth AF, Wan J, Bailey AO, Sun B, Kuchar JA, Green WN, et al. Global analysis of protein palmitoylation in yeast. Cell. 2006;125:1003-13.

9. Nichols CB, Ost KS, Grogan DP, Pianalto K, Hasan S, Alspaugh JA. Impact of protein palmitoylation on the virulence potential of Cryptococcus neoformans. Eukaryot Cell. 2015;14:626-35.

10. Wang C, Chen X, Shi W, Wang F, Du Z, Li X, et al. 2-Bromopalmitate impairs neural stem/progenitor cell proliferation, promotes cell apoptosis and 
induces malformation in zebrafish embryonic brain. Neurotoxicol Teratol. 2015;50:53-63.

11. Hemsley PA, Weimar T, Lilley K, Dupree P, Grierson C. Palmitoylation in plants: new insights through proteomics. Plant Signal Behav. 2013;8:e25209.

12. Zhang YL, Li E, Feng QN, Zhao XY, Ge FR, Zhang Y, et al. Protein palmitoylation is critical for the polar growth of root hairs in Arabidopsis. BMC Plant Biol. 2015;15:50

13. Edmonds MJ. Morgan a. A systematic analysis of protein palmitoylation in Caenorhabditis elegans. BMC Genomics. 2014;15:841.

14. Cho E, Park M. Palmitoylation in Alzheimer's disease and other neurodegenerative diseases. Pharmacol Res. 2016;111:133-51.

15. Young FB, Butland SL, Sanders SS, Sutton LM, Hayden MR. Putting proteins in their place: palmitoylation in Huntington disease and other neuropsychiatric diseases. Prog Neurobiol. 2012;97:220-38.

16. Ducker CE, Stettler EM, French KJ, Upson JJ, Smith CD. Huntingtin interacting protein 14 is an oncogenic human protein: palmitoyl acyltransferase. Oncogene. 2004;23:9230-7.

17. Caballero MC, Alonso AM, Deng B, Attias M, De Souza W, Corvi MM. Identification of new palmitoylated proteins in Toxoplasma gondii. Biochim Biophys Acta. 2016;1864:400-8.

18. Jones ML, Collins MO, Goulding D, Choudhary JS, Rayner JC. Analysis of protein palmitoylation reveals a pervasive role in Plasmodium development and pathogenesis. Cell Host Microbe. 2012;12:246-58,

19. Emmer BT, Nakayasu ES, Souther C, Choi H, Sobreira TJ, Epting CL, et al. Global analysis of protein palmitoylation in African trypanosomes. Eukaryot Cell. 2011;10:455-63.

20. Webb Y, Hermida-Matsumoto L, Resh MD. Inhibition of protein palmitoylation, raft localization, and T cell signaling by 2-bromopalmitate and polyunsaturated fatty acids. J Biol Chem. 2000;275:261-70.

21. Davda D, El Azzouny MA, Tom CT, Hernandez JL, Majmudar JD, Kennedy RT, et al. Profiling targets of the irreversible palmitoylation inhibitor 2-bromopalmitate. ACS Chem Biol. 2013:8:1912-7.

22. Pedro MP, Vilcaes AA, Tomatis VM, Oliveira RG, Gomez GA, Daniotti JL. 2Bromopalmitate reduces protein deacylation by inhibition of acyl-protein thioesterase enzymatic activities. PLoS One. 2013;8:e75232.

23. Alonso AM, Coceres VM, De Napoli MG, Nieto Guil AF, Angel SO, Corvi MM. Protein palmitoylation inhibition by 2-bromopalmitate alters gliding, host cell invasion and parasite morphology in Toxoplasma gondii. Mol Biochem Parasitol. 2012;184:39-43.

24. Maric D, BS MG, Buchanan KT, Olson CL, Emmer BT, Epting CL, Engman DM. Molecular determinants of ciliary membrane localization of Trypanosoma cruzi flagellar calcium-binding protein. J Biol Chem. 2011;286:33109-17.

25. Martins VP, Okura M, Maric D, Engman DM, Vieira M, Docampo R, et al. Acylation-dependent export of Trypanosoma cruzi phosphoinositide-specific phospholipase C to the outer surface of amastigotes. J Biol Chem. 2010;285: 30906-17.

26. Batista CM, Kalb LC, Moreira CM, Batista GT, Eger I, Soares MJ. Identification and subcellular localization of TCHIP, a putative Golgi zDHHC palmitoyl transferase of Trypanosoma cruzi. Exp Parasitol. 2013:134:52-60.

27. Batista CM, Saad F, Ceccoti SPC, Eger I, Soares MJ. Subcellular localization of FLAG taggeg enzymes of the dynamic protein S-palmitoylation cycle of Trypanosoma cruzi epimastigotes. Mem Inst Oswaldo Cruz. 2018;113:10. 1590.

28. Goldston AM, Sharma Al, Paul KS, Engman DM. Acylation in trypanosomatids: an essential process and potential drug target. Trends Parasitol. 2014:30:350-60

29. Herrera L, Brand S, Santos A, Nohara LL, Harrison J, Norcross NR, et al. Validation of n-myristoyltransferase as potential chemotherapeutic target in mammal-dwelling stages of Trypanosoma cruzi. PLoS Negl Trop Dis. 2016;10: e0004540.

30. Contreras VT, Salles JM, Thomas N, Morel CM, Goldenberg S. In vitro differentiation of Trypanosoma cruzi under chemically defined conditions. Mol Biochem Parasitol. 1985;16:315-27.

31. Camargo EP. Growth and differentiation in Trypanosoma cruzi. I. Origin of metacyclic trypanosomes in liquid media. Rev Inst Med Trop São Paulo. 1964;6:93-100

32. Contreras VT, Araújo-Jorge TC, Bonaldo MC, Thomaz N, Barbosa HS, Meirelles MN, et al. Biological aspects of the DM28c clone of Trypanosoma cruzi after metacyclogenesis in chemically defined media. Mem Inst Oswaldo Cruz. 1998;83:123-33.

33. Sambrook J, Fritsch EF, Maniatis T. Molecular cloning - a laboratory manual. 2nd ed. New York: Cold Spring Harbor Laboratory Press; 1989.
34. Chou TC, Martin N. Compusyn for drug combinations: PC software and user's guide. Paramus, NJ, USA: ComboSyn Inc.; 2005. (available at www.combosyn.com)

35. Batista CM, Kessler RL, Eger I, Soares MJ. Trypanosoma cruzi intracellular amastigotes isolated by nitrogen decompression are capable of endocytosis and cargo storage in reservosomes. PLoS One. 2015;10:e0130165.

36. Kessler RL, Soares MJ, Probst CM, Krieger MA. Trypanosoma cruzi response to sterol biosynthesis inhibitors: morphophysiological alterations leading to cell death. PLoS One. 2013;8:e55497.

37. Heytler PG. Uncoupling of oxidative phosphorylation by carbonyl cyanide phenylhydrazones. I. Some characteristics of $\mathrm{m}-\mathrm{Cl}-\mathrm{CCP}$ action on mitochondria and chloroplasts. Biochemistry. 1963;2:357-61.

38. Soares MJ, De Souza W. Endocytosis of gold-labeled proteins and LDL by Trypanosoma cruzi. Parasitol Res. 1991;77:461-8.

39. Schenkman S, Diaz C, Nussenzweig V. Attachment of Trypanosoma cruzi trypomastigotes to receptors at restricted cell surface domains. Exp Parasitol. 1991;72:76-86.

40. Batista CM, Medeiros LC, Eger I, Soares MJ. mAb CZP-315.D9: an antirecombinant cruzipain monoclonal antibody that specifically labels the reservosomes of Trypanosoma cruzi epimastigotes. Biomed Res Int. 2014;2014:714-49.

41. Kalb LC, Frederico YC, Batista CM, Eger I, Fragoso SP, Soares MJ. Clathrin expression in Trypanosoma cruzi. BMC Cell Biol. 2014;15:23.

42. Brown RW, Sharma Al, Engman DM. Dynamic protein S-palmitoylation mediates parasite life cycle progression and diverse mechanisms of virulence. Crit Rev Biochem Mol Biol. 2017;52:145-62.

43. Ceole LF, Gandhi H, Villamizar LH, Soares MJ, O'Sullivan TP. Synthesis of novel quinine analogs and evaluation of their effects on Trypanosoma cruzi. Future Med Chem. 2018;10:391-408.

44. Tyler KM, Engman DM. The life cycle of Trypanosoma cruzi revisited. Int J Parasitol. 2001;31:472-81.

45. Fonseca-Berzal C, Rojas Ruiz FA, Escario JA, Kouznetsov W, Gomez-Barrio A. In vitro phenotypic screening of 7-chloro-4-amino(oxy)quinoline derivatives as putative anti-Trypanosoma cruzi agents. Bioorg Med Chem Lett. 2014;24:1209-13.

46. Kingdoms Protozoa C-ST. Chromista and the eozoan root of the eukaryotic tree. Biol Lett. 2010;6:342-5.

47. Maslov DA, Opperdoes FR, Kostygov AY, Hashimi H. Recent advances in trypanosomatid research: genome organization, expression, metabolism, taxonomy and evolution. Parasitology 2018 14:1-27. In Press. https://doi. org/10.1017/S0031182018000951.

48. Schofield C. Trypanosoma cruzi-the vector-parasite paradox. Mem Inst Oswaldo Cruz. 2000;95:535-44.

49. Coleman RA, Rao P, Fogelsong RJ, ES-G B. 2-brumopalmitoyl-CoA and 2bromopalmitate: promiscuous inhibitors of membrane-bound enzymes. Biochim Biophys Acta. 1992;1125:203-9.

50. Caradonna KL, Engel JC, Jacobi D, Lee CH, Burleigh BA. Host metabolism regulates intracellular growth of Trypanosoma cruzi. Cell Host Microbe. 2013;13:108-17.

51. Michaelson D, Ahearn I, Bergo M, Young S, Philips M. Membrane trafficking of heterotrimeric $\mathrm{G}$ proteins via the endoplasmic reticulum and Golgi. Mol Biol Cell. 2002;13:3294-302.

52. Kathayat RS, Cao Y, Elvira PD, Sandoz PA, Zavalla ME, Springer MZ, Drake LE, Macleod KF, van der Goot G, Dickinson BC. Active and dynamic mitochondrial S-depalmitoylation revealed by targeted fluorescent probes. Nat Commun. 2018:9:334.

53. Alvarez E, Girones N, Davis RJ. Inhibition of the receptor-mediated endocytosis of diferric transferrin is associated with the covalent modification of the transferrin receptor with palmitic acid. J Biol Chem. 1990;265:16644-55.

54. Lima MF, Villalta F. Trypanosoma cruzi receptors for human transferrin and their role. Mol Biochem Parasitol. 1990:38:245-52.

55. Porto-Carreiro I, Attias M, Miranda K, De Souza W, Cunha-e-Silva N. Trypanosoma cruzi epimastigote endocytic pathway: cargo enters the cytostome and passes through an early endosomal network before storage in reservosomes. Eur J Cell Biol. 2000;79:858-69.

56. Soares MJ. The reservosome of Trypanosoma cruzi epimastigotes: an organelle of the endocytic pathway with a role on metacyclogenesis. Mem Inst Oswaldo Cruz. 1999:94:139-41.

57. Ducker CE, Griffel LK, Smith RA, Keller SN, Zhuang Y, Xia Z, Diller JD, Smith CD. Discovery and characterization of inhibitors of human palmitoyl acyltransferases. Mol Cancer Ther. 2006;5:1647-59.

58. Jennings $B C$, Nadolski MJ, Ling Y, Baker MB, Harrison ML, Deschenes RJ, Linder ME. 2-Bromopalmitate and 2-(2-hydroxy-5-nitro-benzylidene)-benzo[b]thiophen3-one inhibit DHHC-mediated palmitoylation in vitro. J Lipid Res. 2009; 50:233-42. 